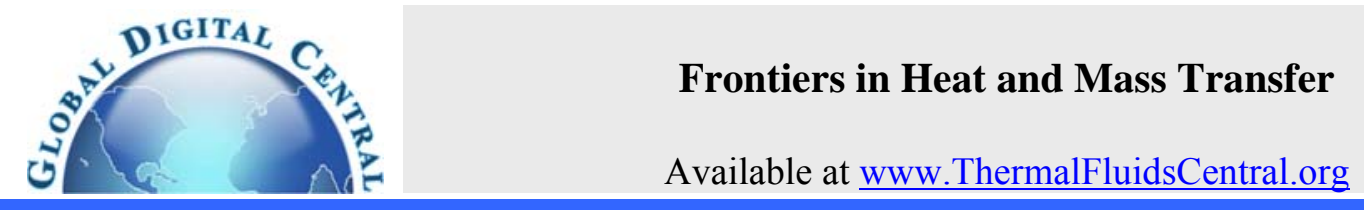

\title{
ENTROPY GENERATION IN BOUNDARY LAYER FLOW OF A MICRO POLAR FLUID OVER A STRETCHING SHEET EMBEDDED IN A HIGHLY ABSORBING MEDIUM
}

\author{
M. Y. Abdollahzadeh Jamalabadi ${ }^{*}$ \\ Chabahar Maritime University, Chabahar 99717-56499, Iran
}

\begin{abstract}
An analytical study of entropy generation in steady boundary layer flow, heat and mass transfer characteristic of $2 D$ convective flow of a micro polar fluid over a stretching sheet embedded through a highly absorbing medium is performed. The governing equations are continuity, momentum boundary layer, micro rotation, and energy takes into account of Rosseland approximation for thermal radiation sources are solved analytically. The governing system of partial differential equations is first transformed into a system of non-linear ordinary differential equations using similarity transformation. The transformed equations are non-linear coupled differential equations which are then linearized by quasi-linearization method and solved very efficiently by the Homotopy analysis method. The special case of the first branch $(K=0$, classical Newtonian fluid) is compared with the existing numerical results of stretching flow in good agreement. In addition, favorable comparisons with previously published work on various special cases of the problem are obtained. The effects of various physical parameters of entropy generation are presented graphically and in tabular form.
\end{abstract}

Keywords: Thermal radiation, boundary layer flow, mixed convection, micro polar fluid, entropy

\section{INTRODUCTION}

Most phenomena in heat and fluid flow are essentially nonlinear and are described by nonlinear equations. Heat transfer in free and mixed convection by simultaneous radiation take place in many applications such as the design of cooling systems for electronic devices (Barletta 1999), solar energy (Barletta 2002), combustion flows (Kandlikar 2005), furnaces (Zhao and $\mathrm{Lu}$ 2002), thermal radiative loading (Abdollahzadeh et al. 2012, Abdollahzadeh et al. 2013, Abdollahzadeh et al. 2014a), and many others (Abdollahzadeh et al. 2014b, Azizah et al. 2013, Shadloo et al. 2015). Furthermore, there is a considerable increase in the research interest in the flows of micro-polar fluids over the past few decades due to the occurrence of these fluids in industrial processes such as exotic lubricants (Khonsari 1990) the flow of colloidal suspensions or polymeric fluids and liquid crystals (Busuke 1969). The boundary layer flow over a continuously stretching surface finds many important applications in engineering processes, such as polymer extrusion and drawing of plastic films, and the applied magnetic field may play an important role in controlling momentum and heat transfers in the boundary layer flow of different fluids over a stretching sheet. Hoyt and Fabula (1964) have shown experimentally that fluids containing minute polymeric additives display a considerable reduction in the skin friction (about $30 \%$ ). The theory of micro polar fluids, which display the effect of local rotary inertia and non-symmetrical stress tensor, was initially introduced and investigated by Eringen (2001), takes into account fluids consisting of randomly oriented particles suspended in a viscous medium. Extensive reviews of the theory and its applications could be found in the review article by Ariman et al. (1973). As well the flow induced by a stretching boundary is important in the extrusion processes in plastic and metal industries.

Recently, extensive attentions have been attracted to the flow induced by a shrinking sheet. Fang et al. (2011) investigated the steady

* Email: my.abdollahzadeh@gnu.ac.kr momentum and heat transfer of a viscous fluid flow over a linearly stretching/shrinking sheet and then a convective boundary condition were further studied analytically. There are some analytic techniques for nonlinear problems, such as perturbation techniques (essentially based on the existence of small or large parameters) that are well known and widely applied. One of the semi-exact methods to solve PDE equations, called the Homotopy Analysis Method (HAM), first proposed by Liao in 1992 in his Ph.D thesis (Liao 1992,Liao 2003). Neither perturbation techniques, nor non-perturbation methods such as the artificial small parameter methods, the $\delta$-expansion method, and Adomian's decomposition method can provide us with a convenient way to adjust and control convergence region and rate of approximation series. This method has already been applied successfully to solve many problems in fluid mechanics (Yun 2013, Rajeev 2014, Yan 2013). In this method, it is possible to adjust and control the convergent region, and this is the most important feature of this technique in comparison to other techniques. In all the previous investigations, the effects of radiation on the flow and heat transfer have not been provided analytically. In the last decade, many researchers have studied the entropy generation in fluid flow and heat transfer over surfaces.

Aiboud and Saouli (2010) presented the application of second law analysis of thermodynamics to viscoelastic flow over a stretching surface. They analytically obtained the velocity and temperature profiles using the Kummer's functions and computed the entropy generation number. Makinde (2011) analyzed the inherent irreversibility in the boundary layer flow of variable viscosity fluid over a semi-infinite flat plate under the influence of thermal radiation. Using local similarity solution technique and shooting quadrature, he numerically obtained the velocity, temperature and entropy generation number. Dehsara et al. (2012) numerically analyzed entropy generation for the mixed convection flow over a nonlinear stretching inclined transparent plate embedded in a porous medium due to solar radiation. Butt et al. (2012a) 
discussed the boundary layer flow and heat transfer analysis of a second grade fluid over a stretching sheet through a porous medium and investigated the effect of viscoelasticity on entropy generation using the Homotopy analysis method (HAM). Butt et al. (2012b) reported the effects of velocity slip on entropy generation in the boundary layer flow over a vertical surface with convective boundary condition. They numerically solved the governing equations using the shooting method and presented expressions for the entropy generation number and Bejan number. Malvandi et al. (2012) analytically studied the steady twodimensional boundary layer flow over an isothermal flat plate by homotopy perturbation method (HPM) and analyzed the entropy generation inside the boundary layer. Galanis and Rashidi (2012) studied the entropy generation in non-Newtonian fluids due to heat transfer in entrance region of ducts. Butt et al. (2012c) made an investigation on entropy generation within a steady laminar mixed convective flow of an over a stretching sheet. They solved the governing equations using the homotopy analysis method and obtained expressions for local entropy generation number, Bejan number, and average Bejan number. Butt and Ali (2013) analyzed the effects of entropy generation in flow over a permeable stretching sheet embedded in a porous medium. They obtained analytical solutions of momentum and energy equations in terms of Kummer's functions and computed the entropy generation number and Bejan number. Noghrehabadi et al. (2013) analyzed the boundary layer heat transfer and entropy generation over an isothermal linearly stretching sheet with heat generation/absorption. They took into account the development of concentration gradient due to slip and the effects of Brownian motion and thermophoresis. Dehsara et al. (2014) investigated the entropy generation of mixed convection flow over a nonlinear stretching inclined transparent plate embedded in a porous medium. Using a numerical algorithm, they solved the governing equations, in the presence of the effects of viscous dissipation, variable magnetic field and solar radiation. More literature survey makes it clear that the entropy generation has not been investigated for the flow and heat transfer of a micropolar fluid over a stretching surface.

Motivated by the works mentioned above, in this paper the HAM is used to find an analytical solution to the velocity and temperature fields with the radiation effect in stretching sheet immersed in micro polar fluid. The purpose of this attempt is to analyze the entropy generation of micropolar flow over a flat plate. Therefore, the local similarity solutions will be found and then the series solution will be first computed using HAM and then its convergence will be discussed in detail. Afterward, the results for the entropy generation profiles will be calculated and discussed from the physical point of view. The entropy generation is calculated using the entropy relation by substituting the velocity and temperature fields obtained from the momentum and entropy equations.

\section{GOVERNING EQUATIONS AND NUMERICAL METHOD}

Consider a steady laminar two-dimensional mixed convection thermal boundary layer flow of an incompressible, electrically conducting, viscous, and incompressible micro polar fluid towards a stretching surface coinciding with the plane $y=0$, the flow region $y>0$. The origin $(\mathrm{x}=0)$ fixed as shown in Fig. 1 . The $\mathrm{x}$-axis is taken in the direction along the sheet and the $\mathrm{y}$-axis is taken perpendicular to it. With these assumptions the governing equations are given by (Nazar 2004) as follow:

The equation of continuity

$$
\frac{\partial \mathrm{u}}{\partial \mathrm{x}}+\frac{\partial v}{\partial \mathrm{y}}=0
$$

The equation of momentum

$$
\mathrm{u} \frac{\partial \mathrm{u}}{\partial \mathrm{x}}+v \frac{\partial \mathrm{u}}{\partial \mathrm{y}}=\left(v+\frac{\kappa}{\rho}\right) \frac{\partial^{2} \mathrm{u}}{\partial \mathrm{y}^{2}}+\frac{\kappa}{\rho} \frac{\partial \mathrm{N}}{\partial \mathrm{y}},
$$

The equation of angular momentum

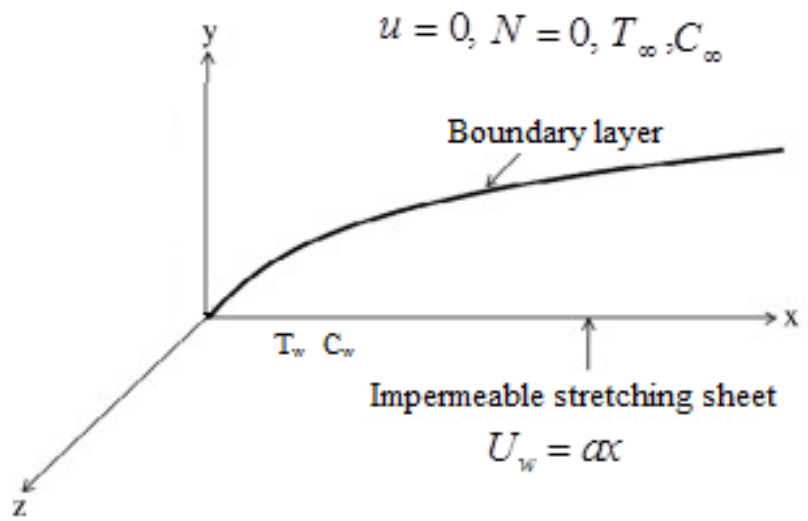

Fig. 1 Schematic of the boundary layer induced by stretching sheet.

$$
\mathrm{u} \frac{\partial \mathrm{N}}{\partial \mathrm{x}}+v \frac{\partial \mathrm{N}}{\partial \mathrm{y}}=\frac{\gamma}{\rho \mathrm{j}} \frac{\partial^{2} \mathrm{~N}}{\partial \mathrm{y}^{2}}-\frac{\kappa}{\rho \mathrm{j}}\left(2 \mathrm{~N}+\frac{\partial \mathrm{u}}{\partial \mathrm{y}}\right),
$$

The equation of energy

$$
\mathrm{u} \frac{\partial \mathrm{T}}{\partial \mathrm{x}}+v \frac{\partial \mathrm{T}}{\partial \mathrm{y}}=\frac{\mathrm{k}}{\rho \mathrm{C}_{\mathrm{P}}} \frac{\partial^{2} \mathrm{~T}}{\partial \mathrm{y}^{2}}-\frac{1}{\rho \mathrm{C}_{\mathrm{P}}} \frac{\partial \mathrm{q}_{\mathrm{r}}}{\partial \mathrm{y}},
$$

The equation of mass diffusion

$$
\mathrm{u} \frac{\partial \mathrm{C}}{\partial \mathrm{x}}+v \frac{\partial \mathrm{C}}{\partial \mathrm{y}}=\mathrm{D} \frac{\partial^{2} \mathrm{C}}{\partial \mathrm{y}^{2}}
$$

where $u$ and $v$ are the velocity components along the $x$ and $y$ directions, $\rho$ is the density of the liquid, $T$ is the temperature of the fluid, $\mathrm{C}_{\mathrm{p}}$ is the specific heat at constant pressure, $v$ is the kinematic viscosity, $\sigma$ is the electrical conductivity of the fluid, $N$ is the components of micro-rotation or angular velocity whose rotation is in the direction of the $x-y$ plane and $\mathrm{j}, \gamma$ and $\kappa$ are the micro inertia per unit mass, spin gradient viscosity and vortex viscosity, respectively. Furthermore, the spin gradient viscosity $\gamma$, which defines the relationship between the coefficient of viscosity and micro inertia as follows (Ahmadi 1976):

$$
\gamma=(\mu+\kappa / 2) \mathrm{j}=\mu(1+\mathrm{K} / 2) \mathrm{j},
$$

where $\mu$ is the dynamic viscosity and $\mathrm{K}=\kappa / \mu$ is the dimensionless viscosity ratio and is called the material parameter. It is worth mentioning here that relation (6) is invoked to allow equations (1)-(4) to predict the correct behavior in the limiting case when microstructure effects become negligible, and the micro rotation, $N$, reduces to the angular velocity (Yücel 1989). It is also worth mentioning that the case $\mathrm{K}=0$ describes the classical Navier-Stokes equations for a viscous and incompressible Newtonian fluid. The flow is generated by the action of two equal and opposite forces along the $\mathrm{x}$-axis and the sheet is stretched in such a way that the velocity at any instant is proportional to the distance from the origin (x-component of the velocity varies linearly along it).

$$
\mathrm{u}(\mathrm{y}=0)=\mathrm{U}_{\mathrm{w}}=\alpha \mathrm{x}
$$

Furthermore sheet kept at constant temperature $\mathrm{T}_{\mathrm{w}}$.

$$
\mathrm{T}(\mathrm{y}=0)=\mathrm{T}_{\mathrm{w}}
$$

which is important parameter throughout the boundary layer (Hadjadj et al. 2015). The other physical boundary conditions for the problem under study are given by:

$$
v(\mathrm{y}=0)=0
$$




$$
\begin{gathered}
\mathrm{N}(\mathrm{y}=0)=-\left.\mathrm{m} \frac{\partial \mathrm{u}}{\partial \mathrm{y}}\right|_{\mathrm{y}=0} \\
\mathrm{C}(\mathrm{y}=0)=\mathrm{C}_{\mathrm{w}}, \\
\mathrm{u}(\mathrm{y}=\infty)=0, \\
\mathrm{~N}(\mathrm{y}=\infty)=0, \\
\mathrm{~T}(\mathrm{y}=\infty)=\mathrm{T}_{\infty}, \\
\mathrm{C}(\mathrm{y}=\infty)=\mathrm{C}_{\infty},
\end{gathered}
$$

where $T_{w}$ is the wall temperature of the fluid and $T_{\infty}$ is the temperature of the fluid far away from the sheet, $\mathrm{C}_{\mathrm{w}}$ is the wall concentration of the solute and $\mathrm{C}_{\infty}$ is the concentration of the solute far away from the sheet and $\mathrm{m}$ is the boundary parameter. It should be mentioned that $m$ is a constant such that $0 \leqslant m \leqslant 1$. The case when $m=0$, is called strong concentration, which indicates $\mathrm{N}=0$ near the wall characterizes concentrated particle flows in which the micro-elements close to the wall surface are unable to rotate. The case when $m=1 / 2$ indicates the disappearing of anti-symmetric part of the stress tensor and means weak concentrations whereas $\mathrm{m}=1$ is used for modelling of turbulent boundary layer flows. This assumption is entreated to permit the field of equations to predict the correct behavior in the limiting case when the microstructure effects become negligible, and the micro rotation $N$, reduces to the angular velocity. Following Rosseland approximation (Brewster 1992) with radiative heat flux $\mathrm{q}_{\mathrm{r}}$ in equation (4) is modeled as:

$$
\mathrm{q}_{\mathrm{r}}=-\frac{4 \sigma^{*}}{3 \mathrm{k}^{*}} \frac{\partial \mathrm{T}^{4}}{\partial \mathrm{y}}
$$

where $\sigma^{*}$ and $\mathrm{k}^{*}$ are the Stephan-Boltzman constant and the mean absorption coefficient, respectively. As the differences within the flow are such that $T^{4}$ can be expressed as a linear function of temperature, $\mathrm{T}^{4}=\mathrm{T}_{\infty}^{4}+4 \mathrm{~T}_{\infty}^{3}\left(\mathrm{~T}-\mathrm{T}_{\infty}\right)+\ldots$, expanding $T^{4}$ in a Taylor series about $T_{\infty}$ and neglecting higher order terms thus,

$$
\mathrm{T}^{4} \approx 4 \mathrm{~T}_{\infty}^{3} \mathrm{~T}-3 \mathrm{~T}_{\infty}^{4} .
$$

By substituting equations (16) and (17) in equation (4), one obtains:

$$
\mathrm{u} \frac{\partial \mathrm{T}}{\partial \mathrm{x}}+v \frac{\partial \mathrm{T}}{\partial \mathrm{y}}=\alpha\left(1+\mathrm{N}_{\mathrm{R}}\right) \frac{\partial^{2} \mathrm{~T}}{\partial \mathrm{y}^{2}}
$$

where $\alpha=\mathrm{k} /\left(\rho \mathrm{C}_{\mathrm{P}}\right)$ is the thermal diffusivity, $\mathrm{N}_{\mathrm{R}}=16 \sigma^{*} \mathrm{~T}_{\infty}^{3} /\left(3 \mathrm{kk}^{*}\right)$ is the radiation parameter (Datti et al. 2004), and $\mathrm{k}$ is the thermal conductivity. Introducing the stream function $\psi(\mathrm{x}, \mathrm{y})$ such that:

$$
\begin{gathered}
u=\frac{\partial \psi}{\partial y}, \\
v=-\frac{\partial \psi}{\partial x},
\end{gathered}
$$
form:

The governing Eqs. (2) and (3) admit a self-similar solution of the

$$
\psi(\mathrm{x}, \mathrm{y})=\left(\nu \mathrm{xU}_{\mathrm{w}}\right)^{0.5} \mathrm{f}(\eta),
$$

where $\mathrm{f}$ is the dimensionless stream function and $\eta$ is the similarity variable:

$$
\eta=\left(\frac{\mathrm{U}_{\mathrm{w}}}{v \mathrm{x}}\right)^{0.5} \mathrm{y}
$$

Note that the definitions of $\psi, u$ and $v$ in the above expressions satisfy the continuity equation (1) identically. Also for micro rotation:

$$
\mathrm{N}=\mathrm{U}_{\mathrm{w}}\left(\frac{\mathrm{U}_{\mathrm{w}}}{v \mathrm{x}}\right)^{0.5} \mathrm{~h}(\eta)
$$

The thermal boundary conditions for solving Eq. (18) depend on the type of heating process considered. Now the non-dimensional temperature and concentration are defined as

$$
\begin{gathered}
\theta(\eta)=\frac{\mathrm{T}-\mathrm{T}_{\infty}}{\mathrm{T}_{\mathrm{w}}-\mathrm{T}_{\infty}} . \\
\phi(\eta)=\frac{\mathrm{C}-\mathrm{C}_{\infty}}{\mathrm{C}_{\mathrm{w}}-\mathrm{C}_{\infty}} .
\end{gathered}
$$

Substituting equations (21) through (25) into the equations (2), (3), (5) and (18), the resulting non-linear third-order ordinary differential equations are:

$$
\begin{gathered}
(1+\mathrm{K}) \mathrm{f}^{\prime \prime \prime}+\mathrm{ff}^{\prime \prime}-\mathrm{f}^{\prime 2}+\mathrm{Kh}=0 \\
\left(1+\frac{\mathrm{K}}{2}\right) \mathrm{h}^{\prime \prime}+\mathrm{f} \mathrm{h}^{\prime}-\mathrm{f}^{\prime} \mathrm{h}-\mathrm{K}\left(2 \mathrm{~h}+\mathrm{f}^{\prime \prime}\right)=0 \\
\frac{1}{\operatorname{Pr}}\left(1+\mathrm{N}_{\mathrm{R}}\right) \theta^{\prime \prime}+\mathrm{f} \theta^{\prime}-2 \mathrm{f}^{\prime} \theta=0 \\
\phi^{\prime \prime}+\mathrm{Sc}\left(\phi^{\prime} \mathrm{f}-2 \phi \mathrm{f}^{\prime}\right)=0
\end{gathered}
$$

where the prime denotes the derivative with respect to the similarity variable (defined in Eq . 22). The Equations (26)-(29) are subject to the following reduced boundary conditions at the wall:

$$
\begin{gathered}
\mathrm{f}(0)=0, \\
\mathrm{f}^{\prime}(0)=1, \\
\mathrm{~h}(0)=-\mathrm{mf}^{\prime \prime}(0), \\
\theta(0)=1, \\
\phi(0)=1,
\end{gathered}
$$

and at the free stream :

$$
\begin{gathered}
\mathrm{f}^{\prime}(\eta=\infty)=0, \\
\mathrm{~h}(\eta=\infty)=0, \\
\theta(\eta=\infty)=0, \\
\phi(\eta=\infty)=0,
\end{gathered}
$$

In the above equations, primes denote differentiation with respect to $\eta$ and $\operatorname{Pr}$ is the dimensionless Prandtl number and is equal to:

$$
\operatorname{Pr}=\frac{\mu \mathrm{C}_{\mathrm{p}}}{\mathrm{k}_{\infty}} .
$$

and also the $\mathrm{Sc}$ is the dimensionless Schmidt number is equal to:

$$
\mathrm{Sc}=\frac{v}{\mathrm{D}} \text {. }
$$

It is noted that $K=0$ is corresponded to viscous fluid case, which is studied before without consideration of the thermal radiation effect 
(Grubka and Bobba 1985). Furthermore It is worth mentioning that when $\mathrm{m}=1 / 2$, one can take $\mathrm{g}(\eta)=-1 / 2 \mathrm{f}^{\prime}(\eta)$, so combining Eqs. (26) and (27) to reduce to a single non-linear ordinary differential equation as

$$
(1+\mathrm{K} / 2) \mathrm{f}^{\prime \prime \prime}+\mathrm{ff}^{\prime \prime}-\mathrm{f}^{\prime 2}=0,
$$

subject to the appropriate boundary conditions

$$
\begin{gathered}
f(0)=0, \\
f^{\prime}(0)=1, \\
f^{\prime}(\eta=\infty)=0,
\end{gathered}
$$

The physical quantities of most interest are the skin friction coefficient $\mathrm{C}_{\mathrm{f}}$ and the local Nusselt number $\mathrm{Nu}_{\mathrm{x}}$, which are defined respectively as:

$$
\begin{gathered}
\mathrm{C}_{\mathrm{f}}=\frac{\tau_{\mathrm{w}}}{\rho \mathrm{U}_{\mathrm{w}}^{2}}, \\
\mathrm{Nu}_{\mathrm{x}}=\frac{\mathrm{xq}_{\mathrm{w}}}{\mathrm{k}\left(\mathrm{T}_{\mathrm{w}}-\mathrm{T}_{\infty}\right)},
\end{gathered}
$$

where the wall shear stress $\tau_{\mathrm{w}}$ and the surface heat flux $\mathrm{q}_{\mathrm{w}}$ for micro polar boundary layer flow are given by:

$$
\begin{gathered}
\tau_{\mathrm{w}}=\left[(\mu+\kappa) \frac{\partial \mathrm{u}}{\partial \mathrm{y}}+\kappa \mathrm{N}\right]_{\mathrm{y}=0}, \\
\mathrm{q}_{\mathrm{w}}=-\mathrm{k}\left(\frac{\partial \mathrm{T}}{\partial \mathrm{y}}\right)_{\mathrm{y}=0},
\end{gathered}
$$

Substituting (26) and (27) into (45) through (48), the following obtain:

$$
C_{\mathrm{f}} \operatorname{Re}_{\mathrm{x}}^{0.5}=(1+K) \mathrm{f}^{\prime \prime}(0)+\mathrm{Kh}(0),
$$

$$
\mathrm{Nu}_{\mathrm{x}} / \mathrm{Re}_{\mathrm{x}}^{0.5}=-\theta^{\prime}(0),
$$

where $\operatorname{Re}_{\mathrm{x}}=\mathrm{U}_{\mathrm{w}} \mathrm{x} / v$ is the local Reynolds number.

\section{APPLICATION OF HOMOTOPY ANALYSIS METHOD}

The governing equations for heat transfer of continuous stretching sheet immersed in a micro polar fluid are expressed by equations (26) through (29). For brevity, the complete operators are defined as follows:

$$
\begin{gathered}
\mathrm{N}_{\mathrm{f}}[\mathrm{f}(\eta ; \mathrm{q})]=(1+\mathrm{K}) \frac{\partial^{3} \mathrm{f}(\eta ; \mathrm{q})}{\partial \eta^{3}}+ \\
\mathrm{K} \frac{\partial \mathrm{h}(\eta ; \mathrm{q})}{\partial \eta}-\left(\frac{\partial \mathrm{f}(\eta ; \mathrm{q})}{\partial \eta}\right)^{2}+\mathrm{f}(\eta ; \mathrm{q}) \frac{\partial^{2} \mathrm{f}(\eta ; \mathrm{q})}{\partial \eta^{2}} \\
\mathrm{~N}_{\mathrm{h}}[\mathrm{h}(\eta ; \mathrm{q})]=\left(1+\frac{\mathrm{K}}{2}\right) \frac{\partial^{2} \mathrm{~h}(\eta ; \mathrm{q})}{\partial \eta^{2}}-\mathrm{K}\left(\frac{\partial^{2} \mathrm{f}(\eta ; \mathrm{q})}{\partial \eta^{2}}+2 \mathrm{~h}(\eta ; \mathrm{q})\right) \\
-\frac{\partial \mathrm{f}(\eta ; \mathrm{q})}{\partial \eta} \mathrm{h}(\eta ; \mathrm{q})+\mathrm{f}(\eta ; \mathrm{q}) \frac{\partial \mathrm{h}(\eta ; \mathrm{q})}{\partial \eta}
\end{gathered}
$$

$$
\begin{aligned}
& \mathrm{N}_{\theta}[\theta(\eta ; \mathrm{q})]=\frac{1}{\operatorname{Pr}}\left(1+\mathrm{N}_{\mathrm{R}}\right) \frac{\partial^{2} \theta(\eta ; \mathrm{q})}{\partial \eta^{2}} \\
& +\mathrm{f}(\eta ; \mathrm{q}) \frac{\partial \theta(\eta ; \mathrm{q})}{\partial \eta} \\
& \quad \mathrm{N}_{\phi}[\phi(\eta ; \mathrm{q})]=\frac{1}{\mathrm{Sc}} \frac{\partial^{2} \phi(\eta ; \mathrm{q})}{\partial \eta^{2}} \\
& +\mathrm{f}(\eta ; \mathrm{q}) \frac{\partial \phi(\eta ; \mathrm{q})}{\partial \eta}-2 \phi(\eta ; \mathrm{q}) \frac{\partial \mathrm{f}(\eta ; \mathrm{q})}{\partial \eta}
\end{aligned}
$$

where $\mathrm{q} \in[0,1]$ is the embedding parameter. As the embedding parameter increases from 0 to 1 , the parameters expressed by $U(\eta ; q)$, $V(\eta ; q), Y(\eta ; q)$ and $Z(\eta ; q)$ vary from the initial guess, $U_{0}(\eta), V_{0}(\eta) Y_{0}(\eta)$, and $Z_{0}(\eta)$, to the exact solution, $U(\eta), V(\eta), Y(\eta)$ and $Z(\eta)$. For $\mathrm{q}=0$

$$
\begin{aligned}
& \mathrm{f}(\eta ; 0)=\mathrm{U}_{0}(\eta), \\
& \mathrm{h}(\eta ; 0)=\mathrm{V}_{0}(\eta), \\
& \theta(\eta ; 0)=\mathrm{Y}_{0}(\eta), \\
& \phi(\eta ; 0)=\mathrm{Z}_{0}(\eta),
\end{aligned}
$$

and at $\mathrm{q}=1$

$$
\begin{aligned}
\mathrm{f}(\eta ; 1) & =\mathrm{U}(\eta), \\
\mathrm{h}(\eta ; 1) & =\mathrm{V}(\eta), \\
\theta(\eta ; 1) & =\mathrm{Y}(\eta), \\
\phi(\eta ; 1) & =\mathrm{Z}(\eta) .
\end{aligned}
$$

As the embedding parameter $q$ increases from 0 to 1 , functions varies from the initial guess to the exact solution. Expanding $f(\eta ; q)$, $\mathrm{h}(\eta ; \mathrm{q}), \quad \theta(\eta ; \mathrm{q})$ and $\phi(\eta ; \mathrm{q})$ in a power series of $\mathrm{q}$ in by Taylor's theorem; Similar to the Blasius which provided a solution in power series, using Taylor series with respect to $q$ results in:

$$
\begin{aligned}
& \mathrm{f}(\eta ; \mathrm{q})=\mathrm{U}_{0}(\eta)+\sum_{\mathrm{m}=1}^{\infty} \mathrm{U}_{\mathrm{m}}(\eta) \mathrm{q}^{\mathrm{m}} \\
& \mathrm{h}(\eta ; \mathrm{q})=\mathrm{V}_{0}(\eta)+\sum_{\mathrm{m}=1}^{\infty} \mathrm{V}_{\mathrm{m}}(\eta) \mathrm{q}^{\mathrm{m}}, \\
& \theta(\eta ; \mathrm{q})=\mathrm{Y}_{0}(\eta)+\sum_{\mathrm{m}=1}^{\infty} \mathrm{Y}_{\mathrm{m}}(\eta) \mathrm{q}^{\mathrm{m}}, \\
& \phi(\eta ; \mathrm{q})=\mathrm{Z}_{0}(\eta)+\sum_{\mathrm{m}=1}^{\infty} \mathrm{Z}_{\mathrm{m}}(\eta) \mathrm{q}^{\mathrm{m}}
\end{aligned}
$$

where,

$$
\begin{aligned}
& \mathrm{U}_{\mathrm{m}}(\eta)=\left.\frac{1}{\mathrm{~m} !} \frac{\partial^{\mathrm{m}} \mathrm{f}(\eta ; \mathrm{q})}{\partial \mathrm{q}^{\mathrm{m}}}\right|_{\mathrm{q}=0}, \\
& \mathrm{~V}_{\mathrm{m}}(\eta)=\left.\frac{1}{\mathrm{~m} !} \frac{\partial^{\mathrm{m}} \mathrm{h}(\eta ; \mathrm{q})}{\partial \mathrm{q}^{\mathrm{m}}}\right|_{\mathrm{q}=0}, \\
& \mathrm{Y}_{\mathrm{m}}(\eta)=\left.\frac{1}{\mathrm{~m} !} \frac{\partial^{\mathrm{m}} \theta(\eta ; \mathrm{q})}{\partial \mathrm{q}^{\mathrm{m}}}\right|_{\mathrm{q}=0}, \\
& \mathrm{Z}_{\mathrm{m}}(\eta)=\left.\frac{1}{\mathrm{~m} !} \frac{\partial^{\mathrm{m}} \phi(\eta ; \mathrm{q})}{\partial \mathrm{q}^{\mathrm{m}}}\right|_{\mathrm{q}=0} .
\end{aligned}
$$

Note that the zero-order deformation equations (63-66) contain the auxiliary parameter and the auxiliary function. Assuming that both auxiliary parameter and the auxiliary function are properly chosen so that the series is convergent at $q=1$.

It is known from Liao (2003) that if the solution series (63-66) converges, where the functions are governed by Equations (26-29) under the definitions (67-69), it must be the solution of Equations (26-29). Therefore According to Liao (2003), it is needed only to focus on the convergence of the solution series by properly choosing of q. Homotopy analysis method can be expressed by many different base functions (Liao 
1992), according to the governing equations; it is straightforward to use a base function in the form of:

$$
\begin{aligned}
& \mathrm{U}(\eta)=\sum_{\mathrm{m}=1}^{\infty} \sum_{\mathrm{p}=1}^{\infty} \mathrm{b}_{\mathrm{pm}} \eta^{\mathrm{p}} \mathrm{e}^{-\mathrm{m} \eta}, \\
& \mathrm{V}(\eta)=\sum_{\mathrm{m}=1}^{\infty} \sum_{\mathrm{p}=1}^{\infty} \mathrm{k}_{\mathrm{pm}} \eta^{\mathrm{p}} \mathrm{e}^{-\mathrm{m} \eta}, \\
& \mathrm{Y}(\eta)=\sum_{\mathrm{m}=1}^{\infty} \sum_{\mathrm{p}=1}^{\infty} \mathrm{d}_{\mathrm{pm}} \eta^{\mathrm{p}} \mathrm{e}^{-\mathrm{m} \eta}, \\
& \mathrm{Z}(\eta)=\sum_{\mathrm{m}=1}^{\infty} \sum_{\mathrm{p}=1}^{\infty} \mathrm{c}_{\mathrm{pm}} \eta^{\mathrm{p}} \mathrm{e}^{-\mathrm{m} \eta},
\end{aligned}
$$

where $b_{p m}, k_{p m}, d_{p m}$ and $c_{p m}$ are the coefficients to be determined, and $m$ is the so-called spatial-scale parameter. When the base function is selected, the auxiliary functions $H_{f}(\eta), H_{h}(\eta), H_{\theta}(\eta)$, and $H_{\varphi}(\eta)$, initial approximations $U_{0}(\eta), V_{0}(\eta), Y_{0}(\eta)$ and $Z_{0}(\eta)$ and the auxiliary linear operators $L_{f}, L_{h}, L_{\theta}$ and $L_{\varphi}$ must be chosen in such a way that the corresponding high-order deformation equations have solutions with the functional form similar to the base functions. It is worth mentioning that the presence of expressions such as $\eta \sin (\mathrm{m \eta})$ prevents the convergence of the analytical solution. This method is referred to as the rule of solution expression (Liao 2003). In the first step the zeroth-order solutions deformation of equations of (26)-(29) are estimated by using HAM. Although the solution series (63-69) given by power functions may be valid in the whole region $\eta \in[0,+\infty)$, it is still an analytic-numerical solution, because the initial condition had to be given by numerical techniques. Their convergence regions are dependent on $\mathrm{q}$, and when $\eta$ is small, a large number of terms are needed to gain an accurate approximation for a large $\eta$. Therefore, they are not efficient solution expressions of Equations (26-29). This is mainly because the base functions defined by (63-69) do not automatically satisfy the boundary conditions at infinity. Choosing exponential function as the base functions to express, the auxiliary linear operators $L_{f}, L_{h}, L_{\theta}$ and $\mathrm{L} \varphi$ are chosen through:

$$
\begin{aligned}
& \mathrm{L}_{\mathrm{f}}[\mathrm{f}(\eta ; \mathrm{q})]=\frac{\partial^{3} \mathrm{f}(\eta ; \mathrm{q})}{\partial \eta^{3}}+\frac{\partial \mathrm{f}(\eta ; \mathrm{q})}{\partial \eta} \\
& \mathrm{L}_{\mathrm{h}}[\mathrm{h}(\eta ; \mathrm{q})]=\frac{\partial^{2} \mathrm{~h}(\eta ; \mathrm{q})}{\partial \eta^{2}}+\frac{\partial \mathrm{h}(\eta ; \mathrm{q})}{\partial \eta} \\
& \mathrm{L}_{\theta}[\theta(\eta ; \mathrm{q})]=\frac{\partial^{2} \theta(\eta ; \mathrm{q})}{\partial \eta^{2}}+\frac{\partial \theta(\eta ; \mathrm{q})}{\partial \eta} \\
& \mathrm{L}_{\phi}[\phi(\eta ; \mathrm{q})]=\frac{\partial^{2} \phi(\eta ; \mathrm{q})}{\partial \eta^{2}}+\frac{\partial \phi(\eta ; \mathrm{q})}{\partial \eta}
\end{aligned}
$$

According to the rule of solution expression, Equations corresponding to the auxiliary linear operators in Equations (75) through (78) result in the following properties:

$$
\begin{gathered}
\mathrm{L}_{\mathrm{f}}\left[\mathrm{C}_{1}+\mathrm{C}_{2} \mathrm{e}^{\eta}+\mathrm{C}_{3} \mathrm{e}^{-\eta}\right]=0, \\
\mathrm{~L}_{\mathrm{h}}\left[\mathrm{C}_{4}+\mathrm{C}_{5} \mathrm{e}^{-\eta}\right]=0, \\
\mathrm{~L}_{\theta}\left[\mathrm{C}_{6}+\mathrm{C}_{7} \mathrm{e}^{-\eta}\right]=0, \\
\mathrm{~L}_{\phi}\left[\mathrm{C}_{8}+\mathrm{C}_{9} \mathrm{e}^{-\eta}\right]=0,
\end{gathered}
$$

According to the rule of solution expression and the initial conditions, the initial approximations $U_{0}, V_{0}, Y_{0}$, and $Z_{0}$, as well as the integral constants, $c_{l}$ through $c_{9}$, are expressed as:

$$
\begin{gathered}
\mathrm{U}_{0}(\eta)=\mathrm{c}_{1}+\mathrm{C}_{2} \mathrm{e}^{\eta}+\mathrm{C}_{3} \mathrm{e}^{-\eta}, \mathrm{c}_{1}=0, \mathrm{c}_{2}=0, \mathrm{c}_{3}=\eta, \\
\mathrm{V}_{0}(\eta)=\mathrm{C}_{4}+\mathrm{C}_{5} \mathrm{e}^{-\eta}, \mathrm{c}_{4}=0, \mathrm{c}_{5}=2 \mathrm{~m}, \\
\mathrm{Y}_{0}(\eta)=\mathrm{C}_{6}+\mathrm{c}_{7} \mathrm{e}^{-\eta}, \mathrm{c}_{6}=0, \mathrm{c}_{7}=1, \\
\mathrm{Z}_{0}(\eta)=\mathrm{c}_{8}+\mathrm{c}_{9} \mathrm{e}^{-\eta}, \mathrm{c}_{8}=0, \mathrm{c}_{9}=1,
\end{gathered}
$$

Let us denote a nonzero auxiliary parameter and $\mathrm{H}(\eta)$ a nonzero auxiliary function. Then one can construct the so-called zero-order deformation equation for any constants $\mathrm{C}_{1}-\mathrm{C}_{9}$. The zero $^{\text {th }}$ order deformation equation and their boundary condition for $f(\eta), h(\eta), \theta(\eta)$, and $\varphi(\eta)$ are constructed as the following equations:

$$
\begin{gathered}
(1-\mathrm{q}) \mathrm{L}_{\mathrm{f}}\left[\mathrm{f}(\eta ; \mathrm{q})-\mathrm{U}_{0}(\eta)\right]=\mathrm{q} \hbar_{\mathrm{f}} \mathrm{H}_{\mathrm{f}}(\eta) \mathrm{N}_{\mathrm{f}}[\mathrm{f}(\eta ; \mathrm{q})] \\
(1-\mathrm{q}) \mathrm{L}_{\mathrm{h}}\left[\mathrm{h}(\eta ; \mathrm{q})-\mathrm{V}_{0}(\eta)\right]=\mathrm{q} \hbar_{\mathrm{h}} \mathrm{H}_{\mathrm{h}}(\eta) \mathrm{N}_{\mathrm{h}}[\mathrm{h}(\eta ; \mathrm{q})] \\
(1-\mathrm{q}) \mathrm{L}_{\theta}\left[\theta(\eta ; \mathrm{q})-\mathrm{Y}_{0}(\eta)\right]=\mathrm{q} \hbar_{\theta} \mathrm{H}_{\theta}(\eta) \mathrm{N}_{\theta}[\theta(\eta ; \mathrm{q})] \\
(1-\mathrm{q}) \mathrm{L}_{\phi}\left[\phi(\eta ; \mathrm{q})-\mathrm{Z}_{0}(\eta)\right]=\mathrm{q} \hbar_{\phi} \mathrm{H}_{\phi}(\eta) \mathrm{N}_{\phi}[\phi(\eta ; \mathrm{q})]
\end{gathered}
$$

subject to the boundary conditions similar to (30)-(38). Those must be the exact solution of Equations (26-29) as long as it is convergent. Note that the solution (87-90) contains three auxiliary parameters $\mathrm{q}, \eta$, and $\hbar$. It should be emphasized that, for any given values of $q$ and $\eta$, we still have the freedom to choose a proper value of the auxiliary parameter $\hbar$ to control and adjust the convergence region and rate of the solution (87$90)$, when necessary. According to the rule of solution expression and from equations (87) through (90), the auxiliary functions $H_{f}(\eta), H_{h}(\eta)$ ), $H_{\theta}(\eta)$ and $H_{\varphi}(\eta)$ can be chosen as follows:

$$
\begin{gathered}
\mathrm{H}_{\mathrm{f}}(\eta)=\eta^{\mathrm{p}} \mathrm{e}^{-\mathrm{m} \eta}, \\
\mathrm{H}_{\mathrm{h}}(\eta)=\eta^{\mathrm{p}} \mathrm{e}^{-\mathrm{m} \eta}, \\
\mathrm{H}_{\theta}(\eta)=\eta^{\mathrm{p}} \mathrm{e}^{-\mathrm{m} \eta} . \\
\mathrm{H}_{\phi}(\eta)=\eta^{\mathrm{p}} \mathrm{e}^{-\mathrm{m} \eta} .
\end{gathered}
$$

the nth-order deformation equations is obtained by differentiating equations (87) through (90), $m$ times, with respect to the embedding parameter $q$ and then setting $q=0$ in the final expression and dividing it by $m !$, are reduced to:

$$
\begin{aligned}
& \mathrm{U}_{\mathrm{m}}(\eta)=\chi_{\mathrm{m}} \mathrm{U}_{\mathrm{m}-1}(\eta)+\mathrm{C}_{1}+\mathrm{C}_{2} \mathrm{e}^{\mathrm{q}}+\mathrm{C}_{3} \mathrm{e}^{-\eta} \\
& \hbar\left(\frac{1}{2} \int_{0}^{\eta} \frac{1}{2} \mathrm{e}_{\mathrm{f}}(\eta) \mathrm{R}_{\mathrm{m}}\left(\mathrm{U}_{\mathrm{m}-1}\right) \mathrm{d} \eta-\right. \\
& \mathrm{V}_{\mathrm{m}}(\eta)=\chi_{\mathrm{m}} \mathrm{V}_{\mathrm{m}-1}(\eta) \mathrm{e}^{\mathrm{q}} \mathrm{R}_{\mathrm{m}}\left(\mathrm{U}_{\mathrm{m}-1}\right) \mathrm{d} \eta \\
& \hbar \int_{0}^{\eta} \int_{0}^{\mu} \mathrm{H}_{\varphi}(\eta) \mathrm{e}^{\eta} \mathrm{R}_{\mathrm{m}}\left(\mathrm{V}_{\mathrm{m}-1}\right) \mathrm{d} \eta \mathrm{d} \mu+\mathrm{C}_{4}+\mathrm{C}_{5} \mathrm{e}^{-\eta}, \\
& \mathrm{Y}_{\mathrm{m}}(\eta)=\chi_{\mathrm{m}} \mathrm{Y}_{\mathrm{m}-1}(\eta)+ \\
& \hbar \int_{0}^{\eta} \int_{0}^{\mu} \mathrm{H}_{\theta}(\eta) \mathrm{e}^{\eta} \mathrm{R}_{\mathrm{m}}\left(\mathrm{Y}_{\mathrm{m}-1}\right) \mathrm{d} \eta \mathrm{d} \mu+\mathrm{C}_{6}+\mathrm{C}_{7} \mathrm{e}^{-\eta} . \\
& \mathrm{Z}_{\mathrm{m}}(\eta)=\chi_{\mathrm{m}} \mathrm{Z}_{\mathrm{m}-1}(\eta)+ \\
& \hbar \int_{0}^{\eta} \int_{0}^{\mu} \mathrm{H} \phi(\eta) \mathrm{e}^{\eta} \mathrm{R}_{\mathrm{m}}\left(\mathrm{Z}_{\mathrm{m}-1}\right) \mathrm{d} \eta \mathrm{d} \mu+\mathrm{C}_{8}+\mathrm{C}_{9} \mathrm{e}^{-\eta} .
\end{aligned}
$$

Equations (95)-(98) is the $m$ th order deformation equation for $f(\eta)$, $h(\eta)$, and $\theta(\eta)$, where

$$
\chi_{\mathrm{m}}=\left\{\begin{array}{ll}
0, & \mathrm{~m} \leq 1 \\
1, & \mathrm{~m}>1
\end{array} .\right.
$$

and :

$$
\begin{aligned}
\mathrm{R}_{\mathrm{m}}\left(\mathrm{U}_{\mathrm{m}-1}\right)= & (1+\mathrm{K}) \frac{\mathrm{d}^{3} \mathrm{U}_{\mathrm{m}-1}(\eta)}{\mathrm{d} \eta^{3}}-\mathrm{K} \frac{\mathrm{dV}_{\mathrm{m}-1}(\eta)}{\mathrm{d} \eta} \\
+ & \sum_{\mathrm{z}=0}^{\mathrm{m}-1} \frac{\mathrm{d} \mathrm{U}_{\mathrm{z}}(\eta)}{\mathrm{d} \eta} \frac{\mathrm{d} \mathrm{U}_{\mathrm{m}-1-\mathrm{z}}(\eta)}{\mathrm{d} \eta}-\sum_{\mathrm{z}=0}^{\mathrm{m}-1} \mathrm{U}_{\mathrm{z}}(\eta) \frac{\mathrm{d}^{2} \mathrm{U}_{\mathrm{m}-1-\mathrm{z}}(\eta)}{\mathrm{d} \eta^{2}} \\
\mathrm{R}_{\mathrm{m}}\left(\mathrm{V}_{\mathrm{m}-1}\right) & =\left(1+\frac{\mathrm{K}}{2}\right) \frac{\mathrm{d}^{2} \mathrm{~V}_{\mathrm{m}-1}(\eta)}{\mathrm{d} \eta^{2}}+\mathrm{K}\left(\frac{\mathrm{d}^{2} \mathrm{U}_{\mathrm{m}-1}(\eta)}{\mathrm{d} \eta^{2}}+2 \mathrm{~V}_{\mathrm{m}-1}(\eta)\right) \\
& \left.-\sum_{\mathrm{z}=0}^{\mathrm{m}-1} \mathrm{~V}_{\mathrm{z}}(\eta) \frac{\mathrm{d} \mathrm{U}_{\mathrm{m}-1-\mathrm{z}}(\eta)}{\mathrm{d} \eta}+\sum_{\mathrm{z}=0}^{\mathrm{m}-1} \mathrm{U}_{\mathrm{z}}(\eta) \frac{\mathrm{dV}_{\mathrm{m}-1-\mathrm{z}}(\eta)}{\mathrm{d} \eta}\right)
\end{aligned}
$$




$$
\begin{aligned}
\mathrm{R}_{\mathrm{m}}\left(\mathrm{Y}_{\mathrm{m}-1}\right)= & \frac{1}{\operatorname{Pr}}\left(1+\mathrm{N}_{\mathrm{R}}\right) \frac{\mathrm{d}^{2} \mathrm{Y}_{\mathrm{m}-1}(\eta)}{\mathrm{d} \eta^{2}}-\sum_{\mathrm{z}=0}^{\mathrm{m}-1} \mathrm{U}_{\mathrm{z}}(\eta) \frac{\mathrm{d} \mathrm{Y}_{\mathrm{m}-1-\mathrm{z}}(\eta)}{\mathrm{d} \eta} \\
& +2 \sum_{\mathrm{z}=0}^{\mathrm{m}-1} \mathrm{Y}_{\mathrm{z}}(\eta) \frac{\mathrm{d} \mathrm{U}_{\mathrm{m}-1-\mathrm{z}}(\eta)}{\mathrm{d} \eta}, \\
\mathrm{R}_{\mathrm{m}}\left(\mathrm{Z}_{\mathrm{m}-1}\right) & =\frac{1}{\mathrm{Sc}} \frac{\mathrm{d}^{2} \mathrm{Z}_{\mathrm{m}-1}(\eta)}{\mathrm{d} \eta^{2}}-\sum_{\mathrm{z}=0}^{\mathrm{m}-1} \mathrm{U}_{\mathrm{z}}(\eta) \frac{\mathrm{d} \mathrm{Z}_{\mathrm{m}-1-\mathrm{z}}(\eta)}{\mathrm{d} \eta} \\
& +2 \sum_{\mathrm{z}=0}^{\mathrm{m}-1} \mathrm{Z}_{\mathrm{z}}(\eta) \frac{\mathrm{d} \mathrm{U}_{\mathrm{m}-1-\mathrm{z}}(\eta)}{\mathrm{d} \eta},
\end{aligned}
$$

The rate of convergence can be increased when suitable values are selected for $m$ and $p$. According to the rule of solution expression, the suitable values for $m$ and $p$ are $\{p=0, m=1\}$. Consequently, the corresponding auxiliary functions were determined as $H_{f}(\eta)=H_{h}(\eta)=$ $H_{\theta}(\eta)=H_{\varphi}(\eta)=e^{-\eta}$. As a result of this selection, the first and second terms of the solution's series are as follows:

$$
\begin{gathered}
\mathrm{U}_{0}(\eta)=\eta \mathrm{e}^{-\eta}, \\
\mathrm{U}_{1}(\eta)=\frac{\mathrm{h}}{12}\left(9 \mathrm{~K}-12 \mathrm{Km}-9 \eta \mathrm{e}^{-\eta}-5 \mathrm{e}^{-\eta}+\right. \\
\left.3 \mathrm{e}^{-\eta} \eta^{2}-9 \mathrm{e}^{-\eta} \mathrm{K} \eta+3 \mathrm{e}^{-\eta} \mathrm{K} \eta^{2}+\cdots\right) \\
\mathrm{Y}_{0}(\eta)=\mathrm{e}^{-\eta}, \\
\mathrm{Y}_{1}(\eta)=\frac{1}{\operatorname{Pr}} 0.0104166 \mathrm{he}^{-\eta}\left(-32 \mathrm{e}^{-\eta}-32 \mathrm{~N}_{\mathrm{R}} \mathrm{e}^{-\eta}+\right. \\
\left.12 \mathrm{e}^{-2 \eta} \eta \operatorname{Pr}+32+32 \mathrm{~N}_{\mathrm{R}}+\cdots\right) \\
\mathrm{V}_{0}(\eta)=2 \mathrm{me}^{-\eta}, \\
\mathrm{V}_{1}(\eta)=-\frac{1}{36} \mathrm{he}^{-\eta}(-18 \mathrm{~m}-81 \mathrm{Km}+8 \mathrm{~K}- \\
\left.24 \mathrm{me}^{-\eta}+36 \mathrm{Kme}^{-\eta}+12 \mathrm{Ke}^{-\eta} \eta+\cdots\right) \\
\mathrm{Z}_{0}(\eta)=\mathrm{e}^{-\eta}, \\
\mathrm{Z}_{1}(\eta)=\frac{1}{\mathrm{Sc}} 0.0104166 \mathrm{he}^{-\eta} \\
\left(12 \mathrm{e}^{-2 \eta} \eta \mathrm{Sc}+\cdots\right)
\end{gathered}
$$

The above Equations are the semi-analytical results of the homotopy analysis method (HAM) to the nonlinear coupled partial differential equations of a boundary layer flow of a micro polar fluid over a stretching sheet embedded in a highly absorbing medium system. Here the concept of the homotopy from topology is employed to generate a convergent series solution for nonlinear systems. This was enabled by utilizing a homotopy-Mclaurin series to deal with the nonlinearities in the system.

\section{ACCURACY AND CONVERGENCE}

The system of coupled non-linear ordinary differential equations (26-29) together with the boundary conditions (30-38) is solved by converting it to an initial value problem. The analytical solution should converge. As pointed out by Liao (2003), the convergence and rate of approximation for the HAM solutions strongly depend on the value of auxiliary parameter h. For simplicity, first the convergence of that is dependent on

$\hbar$ and $q$ is considered. By means of the so-called h-curve, it is straightforward to choose a proper value of $h$ which ensures that the solution series is convergent. It should be noted that the auxiliary parameter $\hbar$, as pointed out by Liao (2003) controls the convergence and accuracy of the solution series. In order to define a region such that the solution series is independent on $\hbar, \hbar$-curves are plotted as shown in Figure 1. The influence of ${ }_{\text {on }}$ on the convergence of $f(0)$ can be investigated by plotting the so-called h-curves of $f(0)$, as shown in Figure 1.The region where the distribution of $\mathrm{f}, \mathrm{f}^{\prime}, \mathrm{f}^{\prime \prime}, \mathrm{h}, \mathrm{h}^{\prime}, \theta, \theta^{\prime}$ versus $\hbar$ is a horizontal line is known as the convergence region for the corresponding function. It is seen that the interval between -0.2 and -0.9 can be chosen as the convergence region. Since the interval for the admissible values of hg corresponds to the line segments nearly parallel to the horizontal axis. For example, it was known that the admissible values for the parameter $h g$ are $-1.7<\mathrm{h}<-0.5$ in the first branch of solution for the stretching case from Fig. 1. In this paper $\hbar=-1.5$. According to Liao (2003), it is needed only to focus on correctly choosing the auxiliary parameter and the spatial-scale parameter so that the solution is convergent. The homotopy-Pad'e technique is applied to accelerate the convergence of $\mathrm{f}^{\prime}(0)$. For given $q$ and $\eta$, by means of plotting the corresponding h-curves of $f(0)$, a negative $\hbar$ with a small enough value of $|\hbar|$ may be chosen to ensure that the corresponding solution series converge.

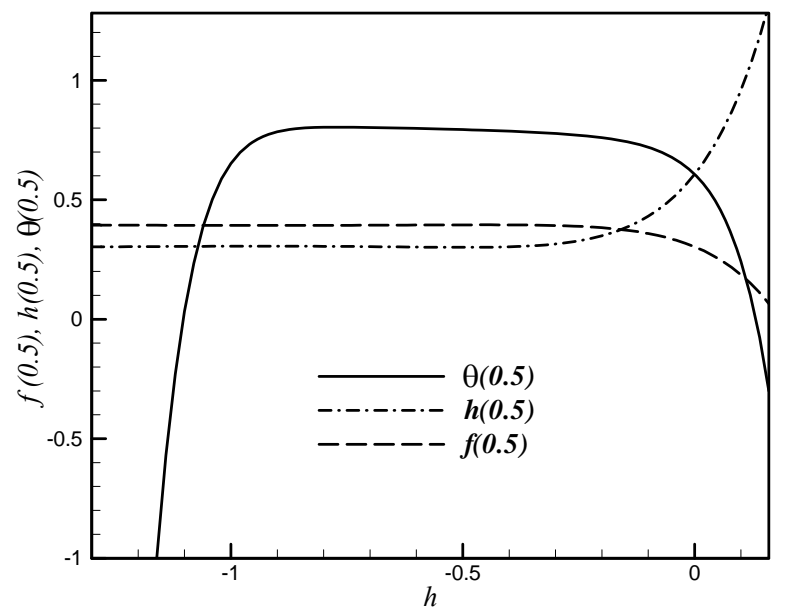

Fig. $2 \hbar$-curves to show the convergence region.

Table 1. Values of $-\mathrm{f}^{\prime \prime}(0)$ for various values of $\boldsymbol{m}$ when $\boldsymbol{K}=\mathbf{0}$.

\begin{tabular}{lll}
$m$ & Cortell (2007) & $\begin{array}{l}\text { Present with } 15^{\text {th }} \\
\text { order }\end{array}$ \\
\hline 0.2 & 0.766758 & 0.766991 \\
0.5 & 0.889477 & 0.889634 \\
1 & 1 & 1 \\
1.5 & 1.061587 & 1.061643 \\
3 & 1.148588 & 1.148635
\end{tabular}

The values of f''(0) for $m<1$ and $m>1$ results in the classical Newtonian fluids case $(\mathrm{K}=0)$ are compared with existing one branch of the numerical results of Cortell (2007) in the case of stretching flow. From the comparison listed in Tables 1, it is found that they are in good agreement. Physically, $\mathrm{f}^{\prime}(0)$ is related to the friction of the fluid on the plate and therefore has important physical meanings. It is found that the $[\mathrm{m}, \mathrm{m}]$ homotopy-Pad'e approximants of $\mathrm{f}$ '( $(0)$ do not depend upon the auxiliary parameter. Besides, the convergence rate of the $[\mathrm{m}, \mathrm{m}]$ homotopy-Pad'e approximants of $\mathrm{f}^{\prime}(0)$ is not sensitive to auxiliary parameter $\eta$, as shown in Table 1. Furthermore in Table 2, a comparison has been made with previous works to show the influence of the iterations order in accuracy. It can be easily seen that, by increasing the number of iterations in series solution, the accuracy increases, which are in very good agreement with other works. Note that the solution (103-110) is explicit, purely analytic, and uniformly valid in the whole region $\eta \in$ $[0,+\infty)$. Thus, it can be regarded as a definition of the solution of Blasius' viscous flow problems governed by Equations (26-29). As shown using the homotopy analysis method, one can obtain many different solution expressions of a nonlinear problem, even if the solution is unique. 
Table 2. Values of $-\theta^{\prime}(0)$ for different $\operatorname{Pr}\left(\mathbf{m}=\mathbf{0 . 5}, N_{R}=\mathbf{0}, K=0\right)$

\begin{tabular}{|c|c|c|c|c|c|}
\hline $\operatorname{Pr}$ & $\begin{array}{l}\text { Grubka and Bobba } \\
(1985)\end{array}$ & Ali (1994) & $\begin{array}{l}\text { Chen } \\
(1998)\end{array}$ & Present with $6^{\text {th }}$ order & Present with $15^{\text {th }}$ order \\
\hline 0.01 & 0.0099 & - & 0.00991 & 0.0089 & 0.00991 \\
\hline 0.72 & 0.4631 & 0.4617 & 0.46315 & 0.4595 & 0.04631 \\
\hline 1 & 0.582 & 0.5801 & 0.58199 & 0.5793 & 0.0582 \\
\hline 3 & 1.1652 & 1.1599 & 1.16523 & 1.16381 & 1.1652 \\
\hline 10 & 2.308 & 2.296 & 2.30796 & 2.2998 & 2.30791 \\
\hline 100 & 7.7657 & - & 7.76536 & 7.7532 & 7.76532 \\
\hline
\end{tabular}

\section{ENTROPY GENERATION}

It is easy to show that the rate of a one-way destruction of useful work in an engineering system $\mathrm{W}_{\text {lost }}$ is directly proportional to the rate of entropy generation:

$$
\mathrm{W}_{\text {lost }}=\mathrm{T}_{0} \dot{\mathrm{S}}_{\mathrm{g}}^{\prime \prime \prime}
$$

where $T_{0}$ is the absolute temperature of the ambient. Assuming a finite size control volume at an arbitrary point in a two dimensional convection flow field and applying the second law of

Thermodynamics, as Bejan (1996) suggested entropy generation per unit time and per unit volume equation is:

$$
\dot{\mathrm{S}}_{\mathrm{g}}^{\prime \prime \prime}=\mathrm{k}\left(\frac{\nabla \mathrm{T}}{\mathrm{T}}\right)^{2}+\frac{\mu}{\mathrm{T}}\left(\left(\frac{\partial \mathrm{u}}{\partial \mathrm{y}}\right)^{2}+\left(\frac{\partial \mathrm{v}}{\partial \mathrm{x}}\right)^{2}\right)
$$

Substituting Eqs. (21), (22) and (24) into Eq. (111) a relation for entropy generation for the flow over a horizontal flat plate is

obtained:

$$
\mathrm{S}_{\text {total }}=\frac{\dot{\mathrm{S}}_{\mathrm{g}}^{\prime \prime \prime}}{\mathrm{S}}=\frac{1}{2 \operatorname{Pr} \operatorname{Re} \mathrm{Ec}}\left(\frac{\theta^{\prime}}{\theta+\theta_{\mathrm{r}}}\right)^{2}\left(\frac{\eta^{2}}{2 \operatorname{Re}}+1\right)+
$$

$$
\frac{1}{2 \operatorname{Re}}\left(\frac{\mathrm{f}^{\prime \prime 2}}{\theta+\theta_{\mathrm{r}}}\right)
$$

where $\mathrm{S}$ is

$$
\mathrm{S}=\frac{\rho^{2} \mathrm{U}_{\mathrm{w}}^{4}}{\mu\left(\mathrm{T}_{\mathrm{w}}-\mathrm{T}_{\infty}\right)}
$$

Temperature ratio is

$$
\theta_{\mathrm{r}}=\frac{\mathrm{T}_{\infty}}{\mathrm{T}_{\mathrm{w}}-\mathrm{T}_{\infty}}
$$

and the Eckert number (Ec) is a dimensionless number which characterize dissipation by express the relationship between a flow's kinetic energy and enthalpy as

$$
\mathrm{Ec}=\frac{\mathrm{U}_{\mathrm{w}}^{4}}{\mathrm{C}_{\mathrm{p}}\left(\mathrm{T}_{\mathrm{w}}-\mathrm{T}_{\infty}\right)}
$$

Equation (112) denotes the entropy generation in terms of similarity parameters as well as similarity functions. So, it is straightforward to calculate the entropy generation with the aid of similarity solution. It must be noted that in Eq. (112) the first term is because of heat transfer and the latter one is due to fluid friction; Bejan number is defined as follows:

$$
\mathrm{Be}=\frac{1}{2 \operatorname{Pr} \operatorname{Re} \mathrm{EcS}_{\text {total }}}\left(\frac{\theta^{\prime}}{\theta+\theta_{\mathrm{r}}}\right)^{2}\left(\frac{\eta^{2}}{2 \mathrm{Re}}+1\right)
$$

As is obvious, $\mathrm{Be}$ yields the share of $\mathrm{S} \mathrm{h}$ and $\mathrm{S} \mathrm{f}$ in total generated entropy, $\mathrm{Be}=1$ is the limit at which the heat transfer irreversibility dominates, $\mathrm{Be}=0$ is the opposite limit at which the irreversibility is dominated by fluid friction effects and $\mathrm{Be}=1 / 2$ is the case that the heat transfer and fluid friction entropy generation rates are equal.

\section{RESULTS AND DISCUSSION ERRORS}

In order to study the effects of governing parameters on the flow and heat transfer characteristics, the graphical presentation of analytical results is presented in this section. Figure 3 presents the $\mathrm{f}$ profiles for various values of $\mathrm{K}(1,10,100$, and 1000$)$ when $\mathrm{m}=0.9, \mathrm{Pr}=1$ and $\mathrm{NR}=1$.From this Figure it is obvious that the $\mathrm{f}$ profiles increase as values of $\mathrm{K}$ increase. By observation of the increase in the curvature, it can also be noticed that the velocity gradient at the surface $f^{\prime \prime}(0)$ decreases (in an absolute sense) as $\mathrm{K}$ increases. The same trend in similar fixed values is seen in Figure 4 for the dimension-less velocity. From this, it can be concluded that micro polar fluids exhibit drag reduction compared to viscous fluids. The negative velocity gradient at the surface, $\mathrm{f}^{\prime \prime}(0)$, as shown in Figure 4 reveals that a drag force is exerted by the stretching sheet on the fluid. This outcome is expected, because the development of the boundary layer is only induced by velocity profile.

Figure 5 shows the variations of the angular velocity profile with respect to $K(5,10,30,50$, and 500) when $\mathrm{m}=0.1, \mathrm{Pr}=1$ and $\mathrm{NR}=1$ parameter. It can be seen that as $\mathrm{K}$ increases, the profiles decrease at the beginning (near the surface) and then (as $\eta$ increases) increase smoothly. The effect of $\mathrm{N}_{\mathrm{R}}$ parameter over the temperature distribution when $\mathrm{m}=$ $1, \operatorname{Pr}=0.7$ and $\mathrm{K}=100$ is represented in Figure 6 . The choice of such high $\mathrm{K}$ is to increase the convective effect and signify the thermal radiation heating. It is clear that the temperature is reduced due to the increase in $\mathrm{K}$ values. This claim is in agreement with the results presented in Table 3, which shows that as the value of $K$ increases, the values of skin friction factors decreases (in an absolute sense) and subsequently a decrease in the temperature profiles occurs. Here the $\mathrm{N}_{\mathrm{R}}$ values are 0,1 , 2, 5, and 10. As revealed in the Figure 6 by increase of $\mathrm{N}_{\mathrm{R}}$ the dimensionless temperature increases. It can be observed that the temperature distribution increases in the flow region as values of $N_{R}$ increase. This is due to the heat energy stored in the liquid because of frictional heating. It is also seen that the temperature gradient at the surface decreases (in an absolute sense) as $\mathrm{N}_{\mathrm{R}}$ increases. This observation is evidence that values of being lower for $N_{R}>0$ compared to $N_{R}=0$ as it presented in Table 4. Thus, the heat transfer rate at the surface is lower in the presence of radiation. However, it can also expect that the heat transfer rate at the surface is increased as $\mathrm{K}$ increases. This observation is in agreement with the results of Table 4

Figure 7 shows a different trend when the effect of $\operatorname{Pr}$ on the temperature distribution was explored as $\mathrm{m}$ and $\mathrm{K}$ values were kept constant at 0.5 and 100 , respectively, and $N_{R}=0$. Here the Pr values are $0,0.05,0.1,0.2,0.3,0.5,1,2$, and 10. From Figure 7 it can be seen that increasing Pr leads to decrease the temperature distribution which 
implies that thermal boundary layer thickness decreases and on the contrary, leads to an increase in the heat transfer rate at the surface.

Figure 8 illustrates the effect of Sc $(0.01,0.1, .3$,and 1$)$ on the dimension-less concentration distribution as $m$ and $K$ values were kept constant at 0.5 and 1 , respectively, and $N_{R}=0$. From Figure 8 it can be concluded that increasing Sc leads to decrease the dimension-less concentration distribution which point toward that concentration boundary layer thickness decreases and on the contrary, leads to an increase in the mass transfer rate at the surface.

Figure 9 presents the $\varphi$ profiles for various values of $K(1,10$, and $100)$ when $\mathrm{m}=0.1, \mathrm{Sc}=0.1$ and $\mathrm{N}_{\mathrm{R}}=1$. From this Figure it is obvious that the $\varphi$ profiles decrease as values of $\mathrm{K}$ increase. By observation of the increase in the curvature, it can also be noticed that the concentration gradient at the surface $c^{\prime \prime}(0)$ increases (in an absolute sense) as $\mathrm{K}$ increases. Such increase on mass convection is important in high Schmitt numbers.

Figure 10 depicted the $\varphi$ profiles for various values of $\mathrm{m}$ $(0,5,0.8,0.9$, and 1$)$ when $\mathrm{K}=1, \mathrm{Sc}=0.1$ and $\mathrm{N}_{\mathrm{R}}=1$. Most of the previous investigations have been done based on considering the boundary parameter $\mathrm{m}=0$ or $\mathrm{m}=0.5(\mathrm{~m}=0$ represents concentrated particle flow in which the elements close to the surface are unable to rotate and $\mathrm{m}=$ 0.5 indicates the vanishing of asymmetric part of the stress tensor). In the present work, more values of $\mathrm{m}$ have been considered. The concentration enhancement effect of boundary parameter $\mathrm{m}$ on concentration profiles is signified in Figure 10. It can also be found that the concentration gradients (mass transfer rate) at surface are larger for larger values of $\mathrm{m}$. Figure 11 presents the f' profiles for various values of $\mathrm{K}(10,50,100$, and 500) when $\mathrm{m}=0.5$. From this Figure it is obvious that the f" profiles increase as values of $\mathrm{K}$ increase. In addition the curvature of $\mathrm{f}$ " decreases (in an absolute sense) as $\mathrm{K}$ increases and it approaches the linear profile shape. The f" values are the significant part in the entropy generation rate that rules the velocity component of entropy $\left(\mathrm{S}_{\mathrm{U}}\right)$.

Figure 12 show the $\theta$ " profiles for various values of $\mathrm{K}(10,50,100$, and 500) when $\mathrm{m}=0.5$.From this Figure it is obvious that the $\theta$ " profiles increase smoothly as values of $\mathrm{K}$ increase. A the $\theta$ " value is the significant part in the entropy generation rate that rules the temperature component of entropy $\left(\mathrm{S}_{\theta}\right)$.

Thermal entropy profiles for various values of Prandtl number $(\mathrm{m}=0.1, \mathrm{~K}=100, \mathrm{NR}=1, \mathrm{Re}=1, \mathrm{r}=0.1$, and $\mathrm{Ec}=1)$ are shown in Figure 13. As shown most of the thermal component of entropy is occurred near the wall and after the $\eta=1$ this contribution is negligible. As well the velocity component of entropy profiles $(\mathrm{m}=0.1, \mathrm{~K}=100, \mathrm{NR}=1, \mathrm{Re}=1$, and $\theta_{\mathrm{r}}=0.1$ ) for various values of Prandtl number are revealed in the Figure 14. As illustrated the maximum of the velocity component of entropy take place at the distance $=1$ from the wall and decrease by distance from this point.

The summation of this components $(\mathrm{m}=0.1, \mathrm{~K}=100, \mathrm{NR}=1, \mathrm{Re}=1$, $\theta \mathrm{r}=0.1$, and $\mathrm{Ec}=1)$ are displayed in the Figure 15 . Total entropy profiles for various values of Prandtl number have maximum near the wall and after the $\eta=1$ are diminishing. To have a better sense from contribution of entropy components the Figure 16 is plotted to show the Bejan number profiles for various values of Prandtl number. By increase of Prandtl number the Bejan number increased near the wall and decrease in the distances far from the wall. The neutral point is take place near the $\eta=1$ point. It must be mentioned that at the beginning of the plate, $\mathrm{Be}$ is approximately unity $(\mathrm{Be} \cong 0.9)$, which means in the total entropy generation, heat transfer plays more important role than fluid friction but as we go along the surface, with decrease in temperature gradient, heat transfer share in total generated entropy decreases.

The thermal boundary layer thickness becomes thicker for stronger Prandtl number. Figure 17 exposed the variation of average Bejan number from $\eta=0$ to $\eta=4$ versus Prandtl number. By increase of $\mathrm{c}$ the average of Bejan number decreased smoothly. Variations of thermal boundary layer thickness and temperature gradients for various values of Prandtl number clearly lead to decrease in Bejan number.

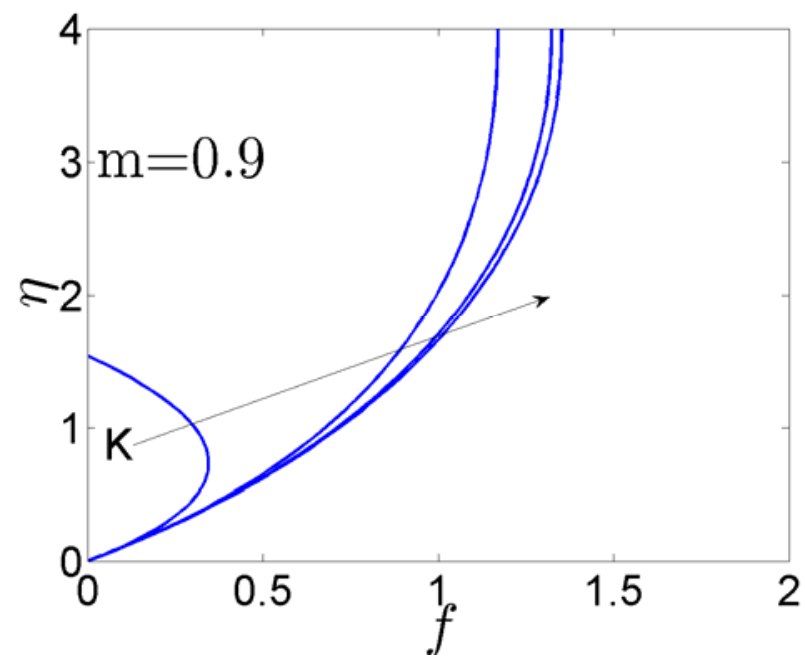

Fig. 3 Variation of $\mathrm{f}(\eta)$ with $\eta$ at different values of $K$.

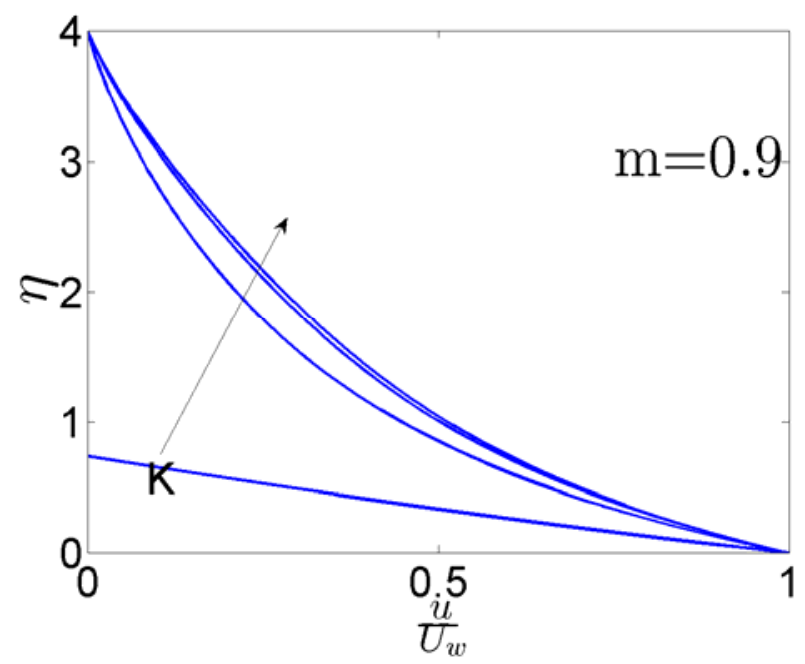

Fig. 4 Variation of velocity profile $\mathrm{f}^{\prime}(\eta)$ with $\eta$ at different values of $K$.

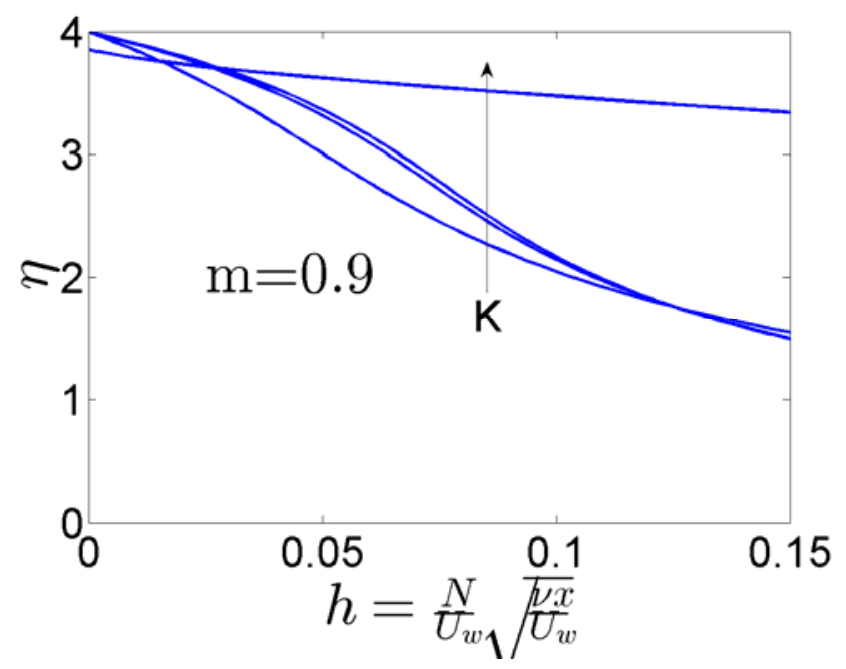

Fig. 5 Angular velocity $h(\eta)$ for various values of $K$. 


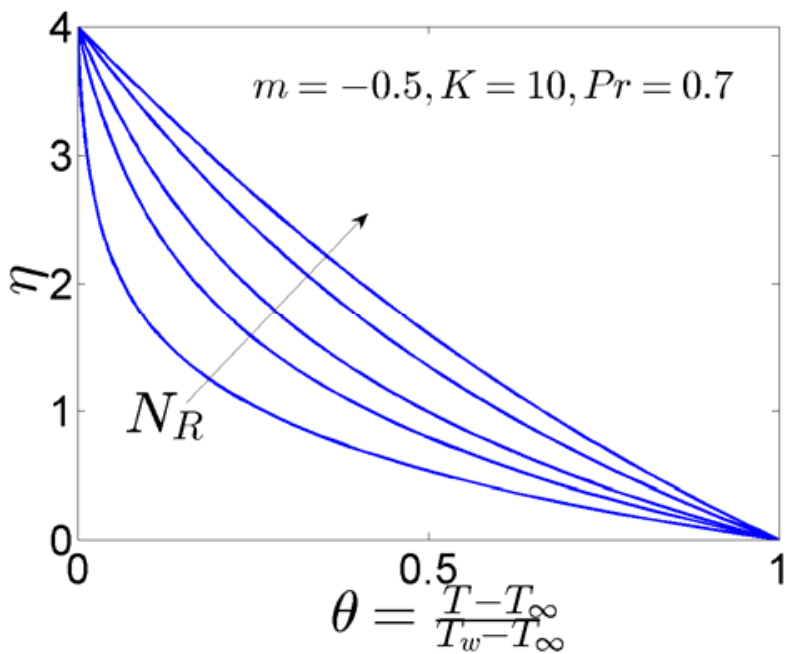

Fig. 6 Temperature profiles $\theta(\eta)$ for various values of $N_{R}$.

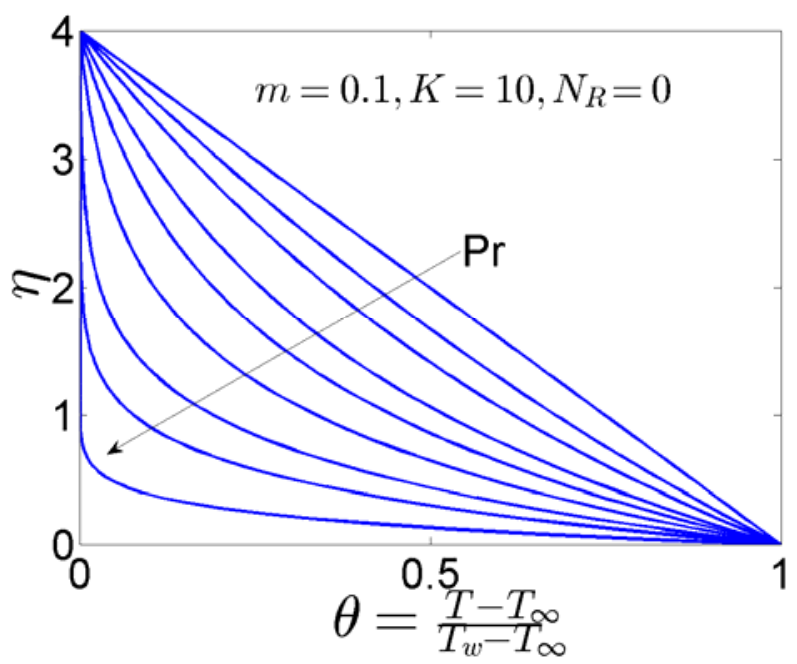

Fig. 7 Temperature profiles $\theta(\eta)$ for various values of $P r$.

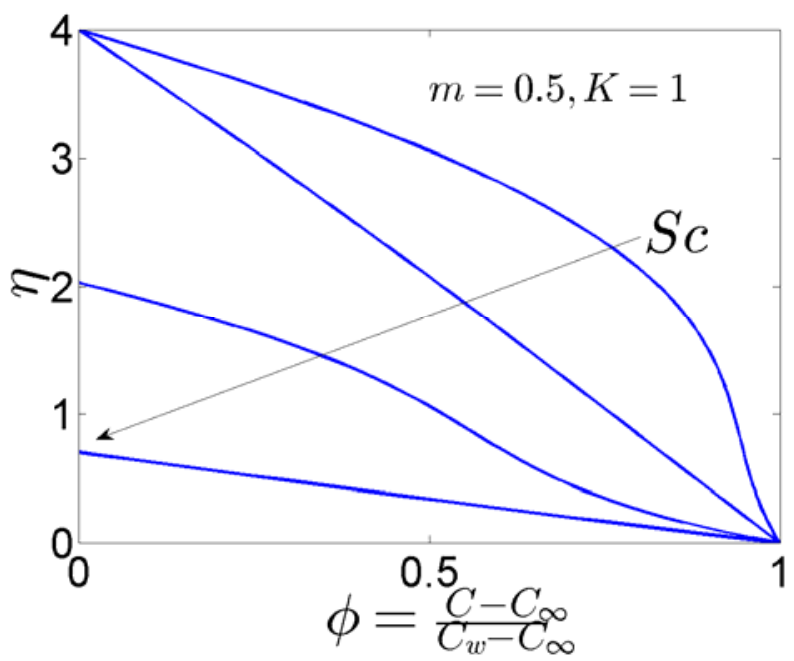

Fig. 8 Concentration profiles $\phi(\eta)$ for various values of $S c$.

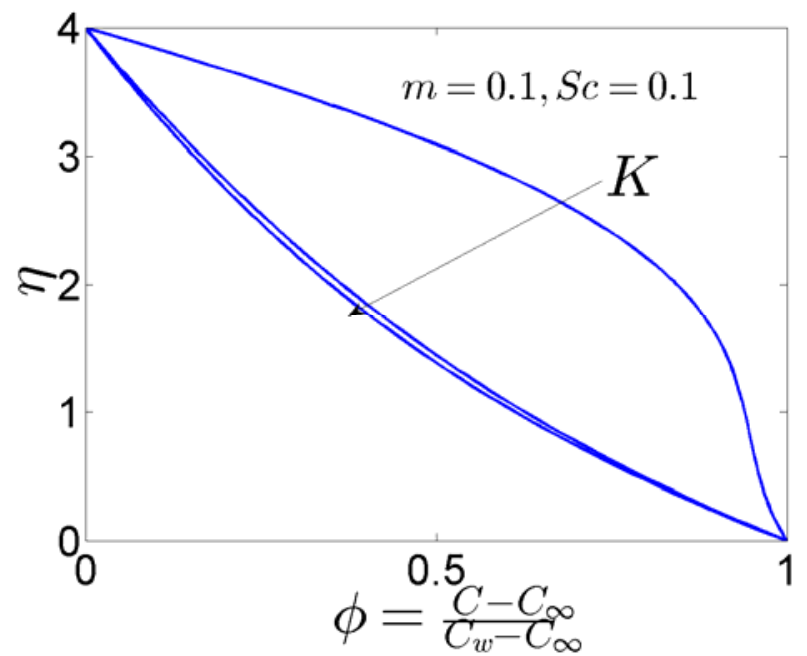

Fig. 9 Concentration profiles $\phi(\eta)$ for various values of $K$.

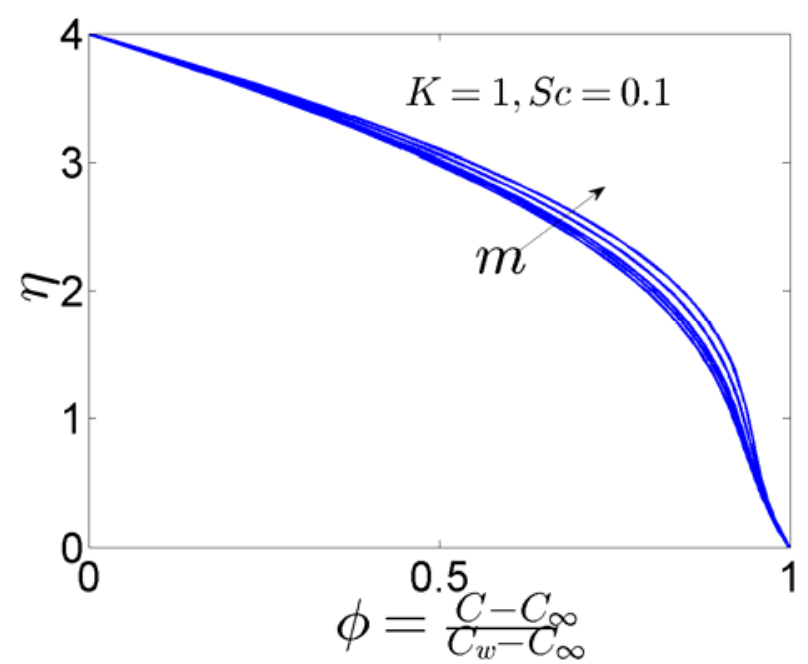

Fig. 10 Concentration profiles $\phi(\eta)$ for various values of $m$.

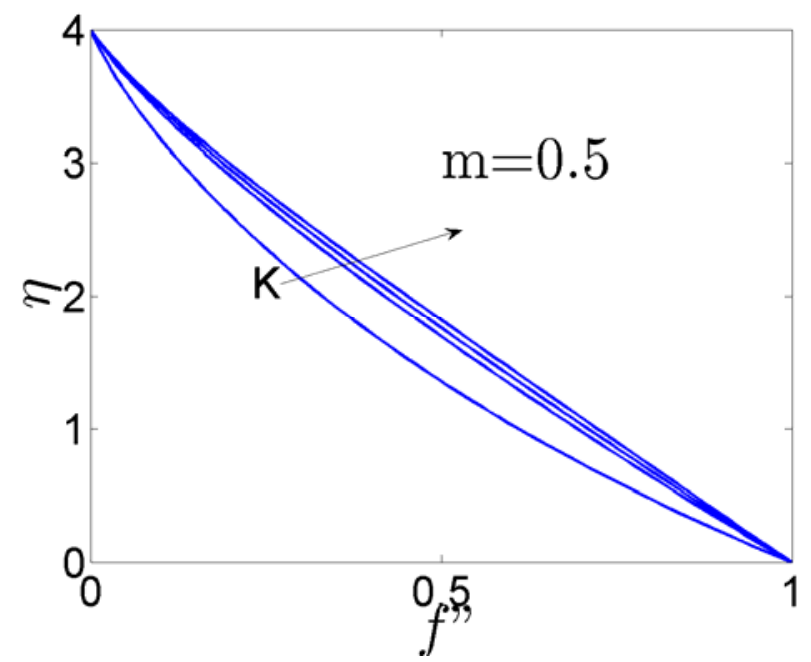

Fig. 11 Variation of velocity profile $\mathrm{f}^{\prime \prime}(\eta)$ with $\eta$ at different values of $K$. 


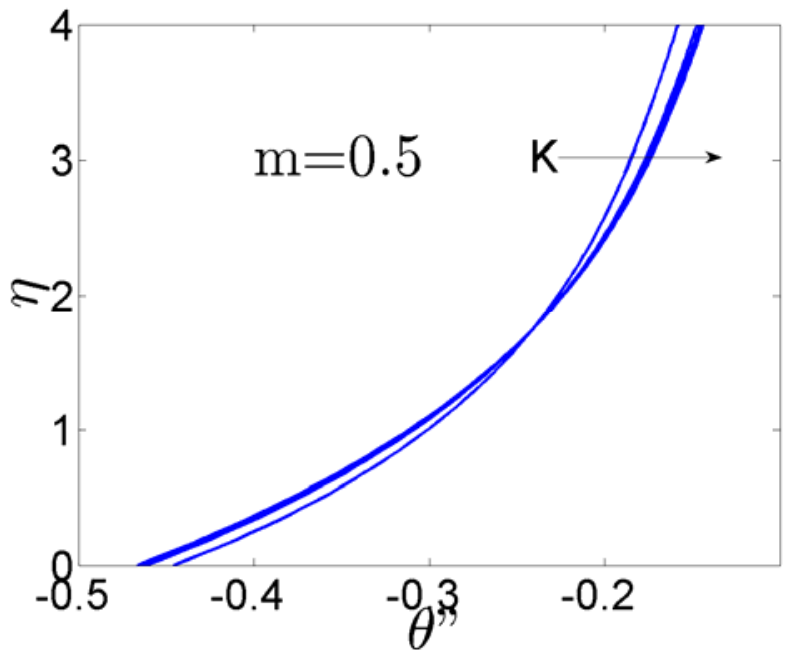

Fig. 12 Variation of velocity profile $\theta^{\prime \prime}(\eta)$ with $\eta$ at different values of K.

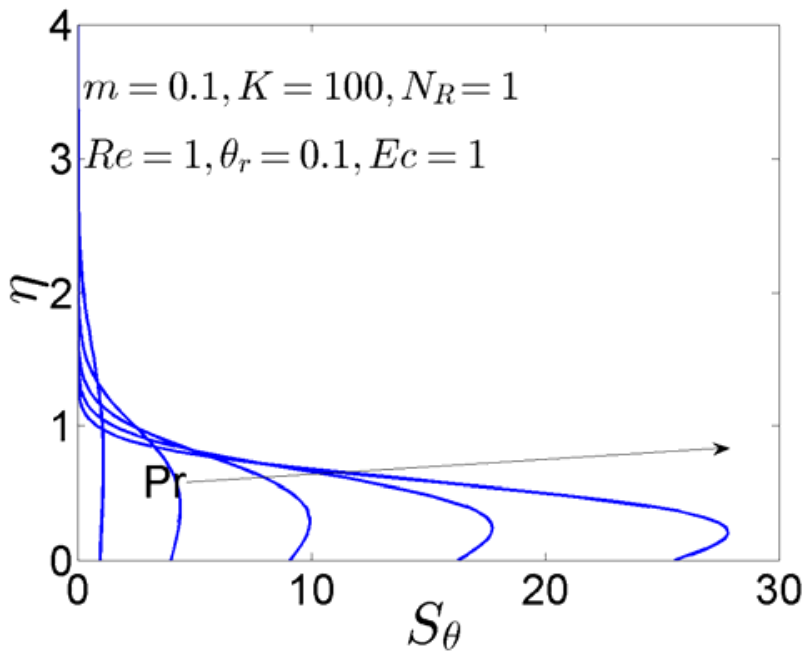

Fig. 13 Thermal entropy profiles $\mathrm{S}_{\theta}(\eta)$ for various values of $P r$.

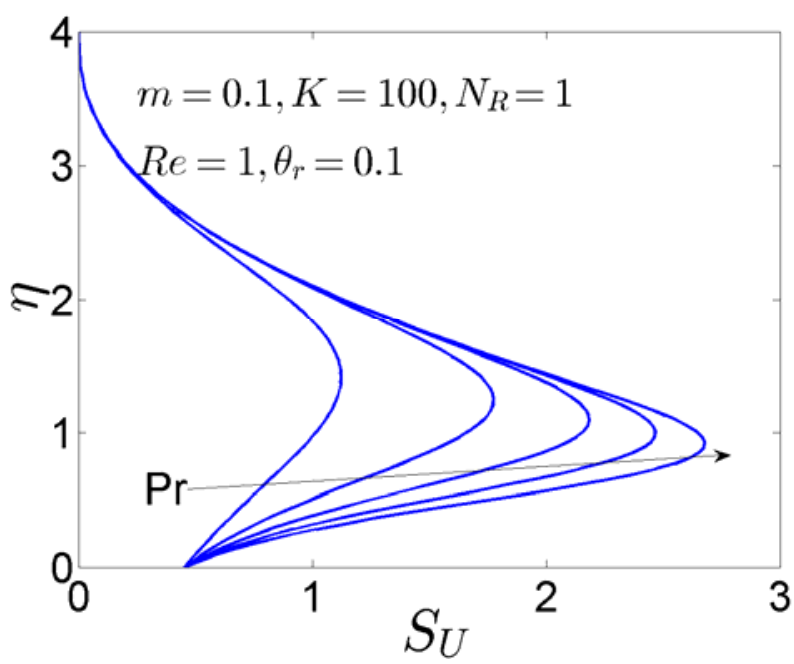

Fig. 14 Velocity entropy profiles $\mathrm{S}_{\mathrm{U}}(\eta)$ for various values of $P r$.

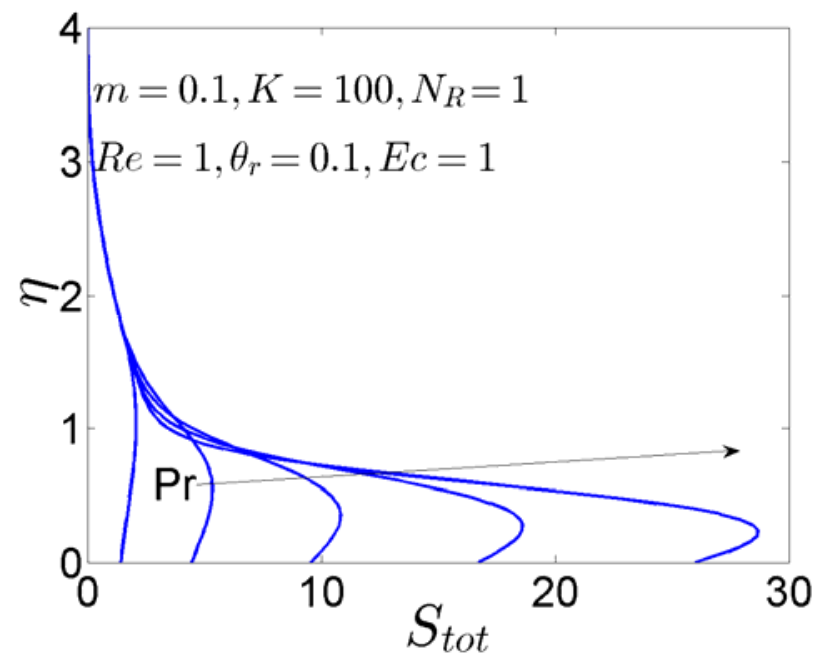

Fig. 15 Total entropy profiles $\mathrm{S}_{\text {tot }}(\eta)$ for various values of $P r$.

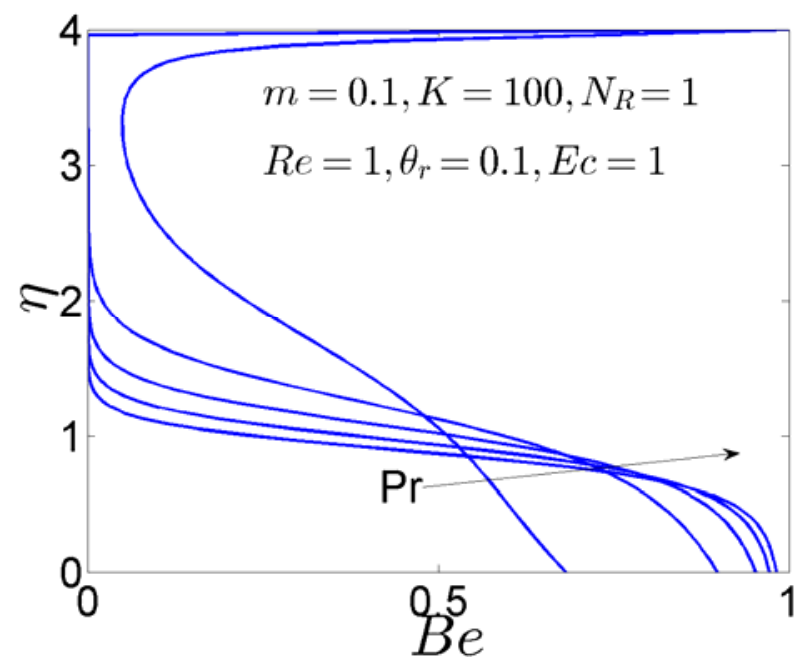

Fig. 16 Bejan number profiles $\operatorname{Be}(\eta)$ for various values of $P r$.

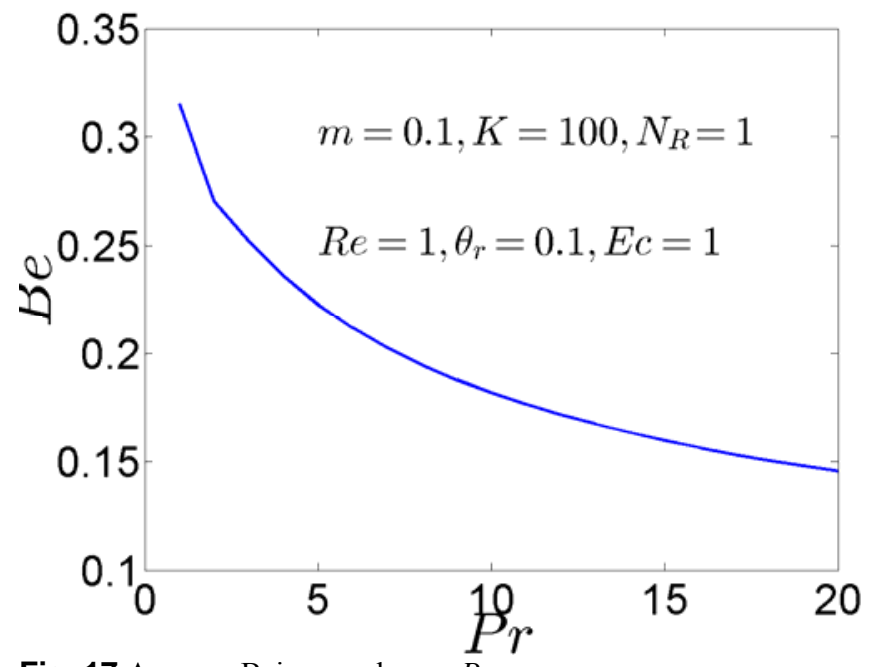

Fig. 17 Average Bejan number vs $P r$. 
Table 3. Values of $-\theta^{\prime}(0)$ for various values of $\operatorname{Pr}, N_{R}, m$ and $K$.

\begin{tabular}{lllll}
$P r$ & $N_{R}$ & $m$ & $K$ & $N u_{x} / \mathrm{Re}_{x}^{0.5}$ \\
\hline & & & 0 & 0.41181 \\
1 & 1 & 0.5 & $\begin{array}{l}1 \\
5\end{array}$ & 0.42723 \\
& & & 5 & 0.47041 \\
& & & 10 & 0.57507 \\
\hline & & 0 & & 0.45513 \\
& & 0.2 & & 0.45217 \\
1 & 1 & 0.4 & 1 & 0.44869 \\
& & 0.6 & & 0.4446 \\
& & 0.8 & & 0.43975 \\
& & 1 & & 0.43378 \\
\hline & 0 & & & 0.61332 \\
1 & 1 & 0.5 & 1 & 0.50777 \\
& 5 & & & 0.42723 \\
& & & & 0.34031 \\
\hline 0.5 & & & & 0.36031 \\
1 & & & & 0.42723 \\
2.5 & 1 & 0.5 & 1 & 0.68679 \\
5 & & & & 0.90555 \\
10 & & & & 1.11815
\end{tabular}

Table 4. Values of Skin friction factor when $P r=1$ and $N_{R}=1$ for various values of $m$ and $K$.

\begin{tabular}{lll}
$\mathrm{m}$ & $\mathrm{K}$ & $C_{f} \mathrm{Re}_{x}^{0.5}$ \\
\hline & 0 & -8.0252 \\
& 1 & -6.5356 \\
0.5 & 2.5 & -5.1566 \\
& 5 & -4.2871 \\
& 10 & -3.3208 \\
\hline 0 & & -10.264 \\
0.2 & & -8.9227 \\
0.4 & 1 & -7.3884 \\
0.6 & & -5.6153 \\
0.8 & & -3.5205 \\
1 & & -1.0118
\end{tabular}

\section{CONCLUSIONS}

In this paper the problem of steady two-dimensional heat transfer of continuous stretching sheet immersed in a micro polar fluid in the existence of radiation have studied. Using existence similarity transformations the governing equations have been transformed into nonlinear ordinary differential equations and solved analytically by using HAM. The obtained results were validated against previous works. Effects of the various parameters such as Prandtl number, the material parameter, boundary parameter and radiation parameter on the temperature, velocity and angular velocity profiles, as well as the skinfriction coefficient and the local Nusselt number were examined. The following conclusions can be drawn as a result of the HAM calculations: -An increase in the value of $K$ leads to a decrease of the angular velocity profiles, the wall temperature, concentration and the skin-friction coefficient; however, the velocity profile and the local Nusselt number are increasing.

-An increase in the value of $m$ leads to an increase of the angular velocity profiles, the wall temperature, concentration, and a decrease of the velocity profile, the local Nusselt number and the skin-friction coefficient.

-An increase in the value of $N_{R}$ leads to a reduction of the local Nusselt number due to the wall temperature enhancement, while the trend is opposite for $\operatorname{Pr}$ number.

-An increase in the value of $S c$ leads to a reduction of the concentration. -Be is plotted and its variation is studied as well. It is found that more entropy generates in boundary layer with increase the Re and Ec while decrease with the increase of Pr numbers.

\section{REFERENCES}

Abdollahzadeh Jamalabadi, M.Y., Ghassemi, M., Hamedi, M.H., 2012, "Two- Dimensional Simulation of Thermal Loading with Horizontal Heat Sources," Proc. Inst. Mech. Eng. C J. Mech. Eng. Sci., 226, 13021308 http://dx.doi.org/10.1177/0954406211419613

Abdollahzadeh Jamalabadi, M.Y., Ghassemi, M., Hamedi, M.H., 2013, "Numerical Investigation of Thermal Radiation Effects on Open Cavity with Discrete Heat Sources," Int. J. Numer Method Heat Fluid Flow, 23, 649-661 http://dx.doi.org/10.1108/09615531311323791

Abdollahzadeh Jamalabadi, M.Y., 2014a, "Experimental Investigation of Thermal Loading of a Horizontal Thin Plate Using Infrared Camera," $J$. King Saud Univ. - Eng. Sci, 26, 159-167. http://dx.doi.org/10.1016/j.jksues.2013.03.002

Abdollahzadeh Jamalabadi, M.Y., Park, J.H., 2014b,"Thermal Radiation, Joule Heating, And Viscous Dissipation Effects on MHD Forced Convection Flow with Uniform Surface Temperature," Open J. Fluid Dyn, 4, 125-132 http://dx.doi.org/10.4236/ojfd.2014.42011

Ahmadi, G., 1976, "Self- Similar Solution Of Incompressible Micropolar Boundary Layer Flow over a Semi-infinite Plate," Int J Eng Sci,14, 639646.

http://dx.doi.org/10.1016/0020-7225(76)90006-9

Aiboud,S., Saouli,S., 2010, "Entropy Analysis For Viscoelastic Magnetohydrodynamic Flow over a Stretching Surface," Int. J. Nonlinear Mech., 45, 482-489

http://dx.doi.org/10.1016/j.ijnonlinmec.2010.01.007

Ali, M.E., 1994, "Heat Transfer Characteristics of a Continuous Stretching Surface", Heat Mass Transfer, 29, 227-234.

http://dx.doi.org/10.1007/BF01539754

Ariman, T., Turka, M.A., Sylvester,N.D., 1973, "Microcontinuum Fluid Mechanics- Review", Int J Eng Sci, 11, 905-930. http://dx.doi.org/10.1016/0020-7225(73)90038-4

Azizah,Y.N., Anuar,I., Ioan,P., 2013, "Hydromagnetic Flow and Heat Transfer Adjacent to a Stretching Vertical Sheet in a Micropolar Fluid," Thermal Science, 17, 525-532 http://dx.doi.org/10.2298/TSCI100308198Y

Barletta, A., 1999, "Analysis of Combine Forced and Free Flow in a Vertical Channel with Viscous Dissipation and Isothermal-Isoflux Boundary Conditions," ASME J. Heat Transfer, 121,349-356. http://dx.doi.org/10.1115/1.2825987 
Barletta, A., 2002, "Fully Developed Mixed Convection and Flow Reversal in a Vertical Rectangular Duct with Uniform Wall Heat Flux," Int. J. Heat Mass Transfer, 45, 641-654.

http://dx.doi.org/10.1016/S0017-9310(01)00160-0

Bejan,A., 1996, Entropy Generation Minimization, (second ed.) CRC, Boca Raton

Brewster, M.Q., 1992, Thermal Radiative Transfer Properties, Wiley, New York

Busuke,H., Tatsuo, T., 1969, "Two-Dimensional Flows of Linear Micropolar Fluids," Int J Eng Sci, 7, 515-522

http://dx.doi.org/10.1016/0020-7225(69)90036-6

Butt,A.S., Unawar,S.M. Mehmood, A., Ali, A. , 2012a, "Effect of viscoelasticity on Entropy Generation in a Porous Medium over a Stretching Plate," World Applied Sciences Journal, 17, 516-523 http://dx.doi.org/10.1.1.390.2183

Butt,A.S., Munawar,S., Ali, A. Mehmood, A., 2012b, "Entropy Generation in Hydrodynamic Slip Flow over a Vertical Plate with Convective Boundary," Journal of mechanical science and technology, 26, 2977-2984

http://dx.doi.org/10.1007/s12206-012-0701-3

Butt,A.S., Munawar,S. , Ali,A., Mehmood, A., 2012c, " Entropy Analysis of Mixed Convective Magnetohydrodynamic Flow of a Viscoelastic Fluid over a Stretching Sheet," Z. Naturforsch. A-J. Phys. Sci., 67, 451-459

http://dx.doi.org/10.5560/ZNA.2012-0055

Butt,A.S., Ali,A., 2013,"Entropy Generation in MHD Flow over a Permeable Stretching Sheet Embedded in a Porous Medium in the Presence of Viscous Dissipation," Int. J. Exergy, 13, 85-101 http://dx.doi.org/10.1504/IJEX.2013.055779

Chen, C.H., 1998, "Laminar Mixed Convection Adjacent to Vertical, Continuously Stretching Sheets", Heat Mass Transfer, 33, 471-476. http://dx.doi.org/10.1007/s002310050217

Cortell, R., 2007, "Viscous Flow and Heat Transfer over a Nonlinearly Stretching Sheet," Appl. Math. Comput. 184, 864-873.

http://dx.doi.org/10.1016/j.amc.2006.06.077

Datti, P.S, Prasadb,K.V., Abelc,M. S., Joshic, A., 2004," MHD Viscoelastic Fluid Flow over a Non-Isothermal Stretching Sheet," Int $J$ Eng Sci, 42, 935-946.

http://dx.doi.org/10.1016/j.ijengsci.2003.09.008

Dehsara, M., Habibi-Matin, M., Dalir, N., 2012, "Entropy Analysis for MHD Flow over a Non-Linear Stretching Inclined Transparent Plate Embedded in a Porous Medium due to Solar Radiation," Mechanika, 18, 524-533

http://dx.doi.org/10.5755/j01.mech.18.5.2694

Dehsara, M., Dalir,N., Nobari, M.R.H., 2014, "Numerical Analysis Of Entropy Generation in Nanofluid Flow over a Transparent Plate in Porous Medium in Presence of Solar Radiation, Viscous Dissipation and Variable Magnetic Field," J. Mech. Sci. Technol., 28, 1819-1831 http://dx.doi.org/10.1007/s12206-014-0329-6

Eringen, A. C., 2001,"Microcontinuum Field Theories. II: Fluent Media," Springer, New York, USA
Fang, T.G.,Yaoa,S., Pop,I., 2011, "Flow and Heat Transfer over a Generalized Stretching/shrinking Wall Problem-Exact Solutions of the Navier-Stokes Equations," Int. J. Non-Linear Mech., 46, 1116-1127 http://dx.doi.org/10.1016/j.ijnonlinmec.2011.04.014

Galanis,N., Rashidi, M.M., 2012, " Entropy Generation in NonNewtonian Fluids due to Heat and Mass Transfer in the Entrance Region of Ducts," Heat Mass Transfer, 48, 1647-1662

http://dx.doi.org/10.1007/s00231-012-1009-7

Grubka, L.J., Bobba, K.M., 1985, "Heat Transfer Characteristics of a Continuous, Stretching Surface with Variable Temperature," ASME J. Heat Transfer, 107, 248-250.

http://dx.doi.org/10.1115/1.3247387

Hadjadj, A., Ben-Nasr, O., Shadloo, M.S., Chaudhuri, A., 2015, "Effect of Wall Temperature in Supersonic Turbulent Boundary Layers: A Numerical Study," International Journal of Heat and Mass Transfer, 81, 426-438

http://dx.doi.org/10.1016/j.ijheatmasstransfer.2014.10.025

Hoyt, J.W., Fabula, A.G., 1964, "The Effect of Additives on Fluid Friction," U.S. Naval ordinance Test station Report, USA.

Kandlikar, S.G., 2005, "High Flux Heat Removal With Microchannels A Roadmap of Challenges and Opportunities," Heat Transfer Eng., 26,514.

http://hdl.handle.net/1850/7413

Khonsari, M.M., 1990, "On the Self-exited Whirl Orbits of a Journal in a Sleeva Bearing Lubricated with Micropolar Fluids," Acta Mechanica, 81, 235

http://dx.doi.org/10.1007/BF01176991

Liao, S.J., 1992, The Proposed Homotopy Analysis Technique for the Solution of Nonlinear Problems, Ph.D. Thesis, Shanghai Jiao Tong University

Liao, S.J., 2003, Beyond Perturbation: Introduction to Homotopy Analysis Method, Boca Raton: Chapman \& Hall/CRC Press

Makinde,O.D., 2011, "Second Law Analysis for Variable Viscosity Hydromagnetic Boundary Layer Flow with Thermal Radiation and Newtonian heating Entropy," 13, 1446-1464 http://dx.doi.org/10.3390/e13081446

Malvandi,A., Ganji, D.D., Hedayati, F., Kaffash, M.H., Jamshidi, M., 2012, "Series Solution of Entropy Generation Toward an Isothermal Flat Plate," Therm. Sci., 16, 1289-1295

http://dx.doi.org/10.2298/TSCI1205289M

Nazar, R., Amina,N., Filipb, D., Pop,I. , 2004, "Stagnation Point Flow of a Micropolar Fluid Towards a Stretching Sheet," Int J Nonlinear Mech,39, 1227-1235.

http://dx.doi.org/10.1016/j.ijnonlinmec.2003.08.007

Noghrehabadi,A., Saffarian, M.R., Pourrajab,R., Ghalambaz,M.,2013, "Entropy Analysis for Nanofluid Flow over a Stretching Sheet in the Presence of Heat Generation/Absorption and Partial Slip," J. Mech. Sci. Technol., 27, 927-937

http://dx.doi.org/10.1007/s12206-013-0104-0

Rajeev, 2014, "Homotopy Perturbation Method for a Stefan Problem with Variable Latent Heat", Thermal Science, 18, 391-398 http://dx.doi.org/10.2298/TSCI110627008R 
Shadloo, M.S., Hadjadj, A., Hussain, F., 2015, "Statistical Behavior of Supersonic Turbulent Boundary Layers with Heat Transfer at $\mathrm{M}_{\infty}=2$," International Journal of Heat and Fluid Flow, 53, 113-134

http://dx.doi.org/10.1016/j.ijheatfluidflow.2015.02.004

Yan, L.M., 2013, "Modified Homotopy Perturbation Method Coupled with Laplace Transform for fractional heat transfer and porous media equations," Thermal Science, 17, 1409-1414 http://dx.doi.org/TSCI1305409Y

Yücel, A., 1989, "Mixed Convection in Micropolar Fluid Flow over a Horizontal Plate with Surface Mass Transfer," Int J Eng Sci, 27, 15931602. http://dx.doi.org/10.1016/0020-7225(89)90153-5

Yun, Y.S.,Chaolu, T., 2013, "Homotopy Perturbation Method for Viscous Heating in Plane Couette Flow," Thermal Science, 17,13551360

http://dx.doi.org/10.2298/TSCI1305355Y

Zhao, C.Y., Lu, T.J., 2002, "Analysis of Microchannel Heat Sinks for Electronics Cooling," Int. J. Heat Mass Transfer 45, 4857-4869. http://dx.doi.org/10.1016/S0017-9310(02)00180-1 